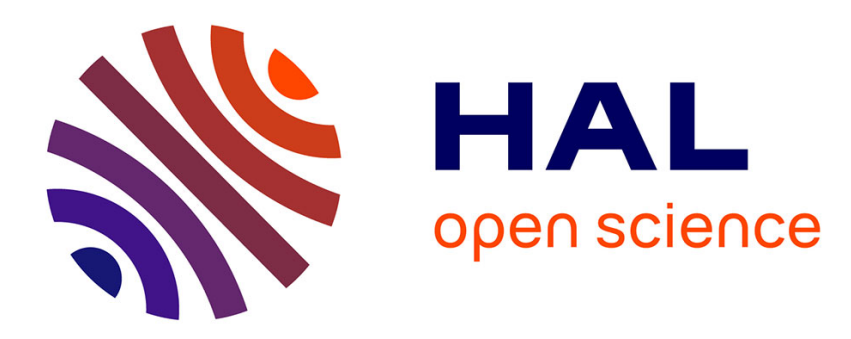

\title{
Two-Way Representations and Weighted Automata Sylvain Lombardy
}

\section{To cite this version:}

Sylvain Lombardy. Two-Way Representations and Weighted Automata. 2015. hal-01187754

\section{HAL Id: hal-01187754 \\ https://hal.science/hal-01187754}

Preprint submitted on 27 Aug 2015

HAL is a multi-disciplinary open access archive for the deposit and dissemination of scientific research documents, whether they are published or not. The documents may come from teaching and research institutions in France or abroad, or from public or private research centers.
L'archive ouverte pluridisciplinaire HAL, est destinée au dépôt et à la diffusion de documents scientifiques de niveau recherche, publiés ou non, émanant des établissements d'enseignement et de recherche français ou étrangers, des laboratoires publics ou privés. 


\title{
Two-Way Representations and Weighted Automata
}

\author{
Sylvain Lombardy \\ Institut Polytechnique de Bordeaux \\ LaBRI, UMR 5800 \\ F-33400 Talence, France \\ sylvain.lombardy@labri.fr
}

\begin{abstract}
We study the series realized by weighted two-way automata, that are strictly more powerful than weighted one-way automata. To this end, we consider the Hadamard product and the Hadamard iteration of formal power series. We introduce the representation of two-way automata and show that the series they realize can be interpreted as the solution of fixed-point equations. In rationally additive semirings, we prove that two-way automata are equivalent to two-way representations, and, for some specific classes of two-way automata, rotating and sweeping automata, we give a characterization of the series that can be realized.
\end{abstract}

\section{Introduction}

Two-way finite automata were introduced at the very beginning of the theory of automata. It was then proved $[16,13]$ that they are not more powerful than one-way automata. Many papers have studied the succintness of two-automata automata with respect to one-way automata ( $c f$. for instance [12]). In this paper, we study weighted two-way automata. This model is strictly more powerful than weighted one-way automata: they have been introduced in [1] where two-way $\mathbb{Z}$-automata that are equivalent to one-way $\mathbb{Z}$-automata have been characterized.

In this paper, we try to characterize the series realized by two-way automata. To this end, we describe different classes of formal power series closed under rational operations, or other operations like the Hadamard product or the mirror.

The definition of weighted two-way automata is quite straighforward from the definition of two-way automata and the definition of weighted one-way automata. Nevertheless, the study of their behaviour is far more complicated: by essence, there may be an infinite number of possible computations on a given input, and it is not easy to state accurate statements for weighted twoway automata without strong assumptions. It is the reason why we delay the study of automata themselves to the end of the paper. We introduce first two-way representations which are algebraic models that are extensions of linear representations. As proved in the last part, they are, under some assumptions on the semiring of weights, equivalent to two-way automata. The set 
of series realizable by such representations, called two-way recognizable series, is closed under sum, Hadamard product, Hadamard iteration, mirror, and left quotient. At the very end of the paper, we use automata to prove that two-way recognizable series are not closed under Cauchy produt and Kleene star.

We then show that two-way recognizable series are solutions of some fixed-point systems. The resolution of such a system would allow to compute an explicit expression for the series realized by a two-way representation. We solve them in the case of rotating and sweeping representations. It proves that rotating representations exactly realize the series which are in the closure of the rational series under sum, Hadamard product and Hadamard iteration; likewise, sweeping representations exactly realize the series which are in the closure of the former set under mirror.

We finally define two-way weighted automata as well as their behaviour. We show that each automaton corresponds to a two-way representation and, in the case of rationally additive semirings, we prove that every two-way weighted automaton is valid and equivalent to its representation.

\section{Formal Power Series}

A semiring is a triple $(\mathbb{K},+,$.$) , where \mathbb{K}$ is a set endowed with two binary associative operations + (addition) and . (multiplication) such that + is commutative and . distributes over $+; \mathbb{K}$ contains two distinct elements 0 and 1 such that 0 is neutral for + and is a annihilator for ., and 1 is neutral for .. Moreover, we assume that every semiring is endowed with a partial unary operation * (star). The semiring $\mathbb{K}$ is commutative if . is commutative.

If $A$ is a finite alphabet of symbols, $A^{*}$ is the set of words over $A$; this set is naturally endowed with the concatenation as multiplication; this operation is associative and admits the empty word $\varepsilon$ (the word with no letter) as neutral element. For every word $w$, we denote $|w|$ the length of $w$, i.e. the number of letters of $w$.

Let $\mathbb{K}$ be a semiring and $A$ be an alphabet. A formal power series $s$ in $\mathbb{K}\left\langle\left\langle A^{*}\right\rangle\right\rangle$ is a mapping from $A^{*}$ to $\mathbb{K}$; for every word $w$, we denote $\langle s, w\rangle$ the image (or coefficient) of $w$ in $s$ and we formally denote $s$ as an infinite sum:

$$
s=\sum_{w \in A^{*}}\langle s, w\rangle w
$$

The support of a formal power series $s$ is the set of words $w$ such that $\langle s, w\rangle$ is different from zero. A series with a finite support is called a polynomial.

\subsection{Rational operations}

Formal power series are extensions of languages. To describe quantitative behaviours, it is natural to extend the regular operations on languages. Let $s$ and $t$ be two formal power series. 
- The sum of $s$ and $t$ is the formal power series

$$
s+t=\sum_{w \in A^{*}}(\langle s, w\rangle+\langle t, w\rangle) w .
$$

- The Cauchy product is

$$
s \cdot t=\sum_{w \in A^{*}}\left(\sum_{u, v=w}\langle s, u\rangle \cdot\langle t, v\rangle\right) w
$$

the unit element for the Cauchy product is the constant series 1.

- The power of a series is inductively defined from the Cauchy product:

$$
s^{k}= \begin{cases}1 & \text { if } k=0 \\ s \cdot s^{k-1} & \text { if } k>0\end{cases}
$$

- The Kleene star of a series is defined in two steps:

- if $s$ is proper $(\langle s, \varepsilon\rangle=0)$,

$$
s^{*}=\sum_{w \in A^{*}}\left(\sum_{n=0}^{\infty}\left\langle s^{k}, w\right\rangle\right) w=\sum_{w \in A^{*}}\left(\sum_{n=0}^{|w|}\left\langle s^{k}, w\right\rangle\right) w ;
$$

- otherwise, $s=s_{0}+s_{p}$, where $s_{0}=\langle s, \varepsilon\rangle$ and $s_{p}$ is proper; in this case, $s^{*}$ exists if and only if $s_{0}^{*}$ exists and

$$
s^{*}=\left(s_{0}^{*} \cdot s_{p}\right)^{*} \cdot s_{0}^{*} .
$$

The sum, the Cauchy product and the Kleene star are called rational operations. The set of rational series $\mathbb{K}$ Rat $A^{*}$ is the smallest set of series which contains polynomials and that is closed under rational operations.

\subsection{Entrywise operations}

The entrywise operations on series are:

- the sum, which is also a rational operation;

- the entrywise product on series, also called Hadamard product: if $s$ and $t$ are two formal power series, then the Hadamard product of $s$ and $t$ is

$$
s \odot t=\sum_{w \in A^{*}}(\langle s, w\rangle \cdot\langle t, w\rangle) w
$$

the unit element for this product is the characteristic series of $A^{*}, 1_{\odot}=\sum_{w \in A^{*}} w$; 
- the Hadamard iteration of a series $s$ in $\mathbb{K}\left\langle\left\langle A^{*}\right\rangle\right\rangle$, which exists if and only if the star of every coefficient of $s$ exists, and

$$
s^{\circledast}=\sum_{w \in A^{*}}\langle s, w\rangle^{*} w .
$$

The set of polynomials is closed under entrywise operations: these operations do not give access to infinity. We can consider two different families of formal power series:

- $\mathbb{K} \mathrm{Had} A^{*}$ is the smallest set of series which contains rational series and that is closed under entrywise operations.

- $\mathbb{K R H} A^{*}$ is the smallest set of series which contains polynomials and that is closed under rational and entrywise operations.

EXAMPLE. Let $A=\{a, b\}$, and let $\mathbb{K}=\mathcal{P}\left(\{a, b\}^{*}\right)$ be the semiring of languages on the alphabet $\{a, b\}$. Let $P=\{a\} a+\{b\} b . S_{1}=P^{*}$ is the rational series identity, where the coefficient of the word is the singleton that contains the word itself.

The series $S_{2}=\left(P^{*}\right) \odot\left(P^{*}\right)$ maps every word onto its square; for instance $\left\langle\left(P^{*}\right) \odot\left(P^{*}\right), a b a\right\rangle=$ $\{a b a a b a\}$. This series of $\mathbb{K} \mathrm{Had} A^{*}$ is not rational.

The series $\left((\{a\} a)^{*} \odot(\{b\} a)^{*}\right) \cdot\left((\{b\} a)^{*} \odot(\{a\} a)^{*}\right)$ maps every word $a^{n}$ onto $\left\{a^{k} b^{n} a^{n-k} \mid k \in\right.$ $[0 ; n]\}$. This series is in $\mathbb{K R H} A^{*} \backslash \mathbb{K H a d} A^{*}$.

\subsection{Mirror operation}

The mirror of a word $w=w_{1} \ldots w_{n}$ is the word $\bar{w}=w_{n} \ldots w_{1}$. This operation can be extended to series: the mirror of a series $s$ is $\bar{s}=\sum_{w \in A^{*}}\langle s, w\rangle \bar{w}$.

Lemma 1. If the semiring $\mathbb{K}$ is commutative, for every alphabet $A$, $\mathbb{K}$ Rat $A^{*}$ is closed under mirror.

The proof is left to the reader; it is very easy to modify a weighted automaton or a rational expression to represent the mirror of a series without increasing the size of the representation.

Lemma 2. The mirror commutes with the entrywise operations on series.

Proof. Let $s$ and $t$ be two series in $\mathbb{K}\left\langle\left\langle A^{*}\right\rangle\right\rangle$. Then

$$
\begin{aligned}
\overline{s+t}=\sum_{w \in A^{*}}\langle s+t, w\rangle \bar{w} & =\sum_{w \in A^{*}}(\langle s, w\rangle+\langle t, w\rangle) \bar{w}=\sum_{w \in A^{*}}\langle s, w\rangle \bar{w}+\sum_{w \in A^{*}}\langle t, w\rangle \bar{w}=\bar{s}+\bar{t} . \\
\overline{s \odot t}=\sum_{w \in A^{*}}\langle s \odot t, w\rangle \bar{w} & =\sum_{w \in A^{*}}(\langle s, w\rangle \cdot\langle t, w\rangle) \bar{w}=\sum_{w \in A^{*}}\langle s, w\rangle \bar{w} \odot \sum_{w \in A^{*}}\langle t, w\rangle \bar{w}=\bar{s} \odot \bar{t} . \\
\overline{s^{\circledast}} & =\sum_{w \in A^{*}}\left\langle s^{\circledast}, w\right\rangle \bar{w}=\sum_{w \in A^{*}}\langle s, w\rangle^{*} \bar{w}=\bar{s}^{\circledast} .
\end{aligned}
$$


Lemma 3. If the semiring $\mathbb{K}$ is commutative, the mirror anticommutes with the Cauchy product and commutes with the Kleene star of series with coefficients in $\mathbb{K}$.

Proof. Let $s$ and $t$ be two series in $\mathbb{K}\left\langle\left\langle A^{*}\right\rangle\right\rangle$. Then

$$
\begin{aligned}
\overline{s \cdot t} & =\sum_{w \in A^{*}}\langle s \cdot t, w\rangle \bar{w}=\sum_{w \in A^{*}}\left(\sum_{u v=w}\langle s, u\rangle \cdot\langle t, v\rangle\right) \overline{u v} \\
& =\sum_{w \in A^{*}}\left(\sum_{u v=w}\langle t, v\rangle \cdot\langle s, u\rangle\right) \bar{v} \bar{u}=\bar{t} \cdot \bar{s} ; \\
\overline{s^{*}} & =\sum_{w \in A^{*}}\left(\sum_{k \in \mathbb{N}, u_{1} \ldots u_{k}=w}\left\langle s, u_{1}\right\rangle \ldots\left\langle s, u_{k}\right\rangle\right) \overline{u_{1} \ldots u_{k}} \\
& =\sum_{w \in A^{*}}\left(\sum_{k \in \mathbb{N}, u_{1}^{\prime} \ldots u_{k}^{\prime}=\bar{w}}\left\langle s, \overline{u_{1}}\right\rangle \ldots\left\langle s, \overline{u_{k}}\right\rangle\right) u_{1}^{\prime} \ldots u_{k}^{\prime}=\bar{s}^{*} .
\end{aligned}
$$

Proposition 4. If the semiring $\mathbb{K}$ is commutative, $\mathbb{K R a t} A^{*}, \mathbb{K H a d} A^{*}$, and $\mathbb{K R H} A^{*}$ are closed under mirror.

If the semiring $\mathbb{K}$ is not commutative and the alphabet $A$ contains at least two letters, the following famillies are different from the ones defined above:

- $\mathbb{K} M i r R a t A^{*}$ is the closure of $\mathbb{K} \operatorname{Rat} A^{*}$ by mirror;

- $\mathbb{K} \operatorname{MirHad} A^{*}$ is the closure of $\mathbb{K} \operatorname{Had} A^{*}$ by mirror;

- $\mathbb{K} \operatorname{MirRH} A^{*}$ is the the closure of $\mathbb{K R H} A^{*}$ by mirror.

\section{Two-Way Representations}

\subsection{A new product of matrices}

In this part, we extend the definitions made in [3] for two-way finite automata.

In one-way $\mathbb{K}$-automata (without $\varepsilon$-transitions), the transition matrix $M$ can be considered as a representation of paths labeled by words of length 1 , and, for every $k, M^{k}$ is the matrix of paths labeled by words of length $k$. The star of the matrix gives then access to the description of the behaviour of the $\mathbb{K}$-automata on every word. If $M$ is the transition matrix of a $\mathbb{K}$-automaton, if $I$ and $T$ are the initial and final vector, the series realized by the automaton is $I . M^{*} . T$. Since the entries of $M^{*}$ are computable (as rational expressions), this is the foundation of the conversion of $\mathbb{K}$-automata into rational expressions.

For two-way automata, the situation is a bit more complicated and a specific product on $\mathbb{K}$-matrices with size $m+n$, must be introduced. 
Such a matrix $M$ is divided into four blocks:

$$
M=\left[\begin{array}{c|c}
\vec{M} \in \mathbb{K}^{m \times m} & \overleftrightarrow{M} \in \mathbb{K}^{m \times n} \\
\hline \overleftrightarrow{M} \in \mathbb{K}^{n \times m} & \overleftarrow{M} \in \mathbb{K}^{n \times n}
\end{array}\right]
$$

Intuitively, such a matrix represents two-way computations on a given word. The upper blocks represents computations that start at the first position of the word, and the lower blocks computations that start at the last position of the word; the left blocks represents computations that end at the last position of the word, and right blocks represents computations that end at the first position of the word. We define now a product of matrices that reflects the computations on the concatenation of two words.

To define this product with reasonable properties (like associativity, for instance), we need some assumptions on the semiring of entries. These identites were introduced in [7].

Definition 1. A semiring $\mathbb{K}$ is a Conway semiring if there exists a star operator defined for every element such that:

$$
\forall x, y \in \mathbb{K}, \quad(x \cdot y)^{*}=1+x \cdot(y \cdot x)^{*} \cdot y, \quad \text { and }(x+y)^{*}=x^{*} \cdot\left(y \cdot x^{*}\right)^{*}=\left(x^{*} \cdot y\right)^{*} \cdot x^{*} .
$$

If $\mathbb{K}$ is a Conway semiring, for every positive integer $n$, the semiring of $\mathbb{K}$-matrices with size $n$ can be endowed with a star operation. If $n=1, M^{*}=\left[M_{1,1}^{*}\right]$, otherwise, for every decomposition

$$
M=\left[\begin{array}{c|c}
X & Y \\
\hline Z & T
\end{array}\right]
$$

where $X$ and $T$ are square matrix with positive sizes, the matrix

$$
\left[\begin{array}{c|c}
\left(X+Y . T^{*} \cdot Z\right)^{*} & \left(X+Y \cdot T^{*} \cdot Z\right)^{*} \cdot Y \cdot T^{*} \\
\hline\left(T+Z \cdot X^{*} \cdot Y\right)^{*} \cdot Z \cdot X^{*} & \left(T+Z \cdot X^{*} \cdot Y\right)^{*}
\end{array}\right]
$$

does not depend on the decomposition and is set as the star of $M$. Then, the semiring $\mathbb{K}^{n \times n}$ is also Conway ( $c f$. [4]).

If $M$ and $N$ are two matrices in $\mathbb{K}^{m+n}$, we set:

$$
M \text { г } N=\left[\begin{array}{c|c}
\vec{M} \cdot(\overleftrightarrow{N} \cdot \overleftrightarrow{M})^{*} \cdot \vec{N} & \overleftrightarrow{M}+\vec{M} \cdot(\overleftrightarrow{N} \cdot \overleftrightarrow{M})^{*} \cdot \overleftrightarrow{N} \cdot \overleftarrow{M} \\
\hline \overleftrightarrow{N}+\overleftarrow{N} \cdot \overleftrightarrow{M} \cdot(\overleftrightarrow{N} \cdot \overleftrightarrow{M})^{*} \cdot \vec{N} & \overleftarrow{N} \cdot(\overleftrightarrow{M} \cdot \overleftrightarrow{N})^{*} \cdot \overleftarrow{M}
\end{array}\right]
$$

This product depends on the pair $(m, n)$, and not only on $m+n$; if needed, it can be explicitely precised as $M \mathbf{2}_{n}^{m} N$.

To prove the associativity, we also need an identity on non square matrices. 
Lemma 5. Let $\mathbb{K}$ be a conway semiring and let $m$ and $n$ be to nonnegative integers. If $M$ is in $\mathbb{K}^{m \times n}$ and $N$ is in $\mathbb{K}^{n \times m}$, then

$$
(M \cdot N)^{*}=\mathrm{Id}_{m}+M \cdot(N \cdot M)^{*} \cdot N .
$$

Proof. If $m=n$, the property holds. If $m>n$, let $M^{\prime}$ and $N^{\prime}$ be two matrices in $\mathbb{K}^{m \times m}$ :

$$
M^{\prime}=\left[\begin{array}{l|l}
M & 0
\end{array}\right], \quad N^{\prime}=\left[\begin{array}{c}
N \\
\hline 0
\end{array}\right]
$$

Then,

$$
M^{\prime} . N^{\prime}=M \cdot N, \quad N^{\prime} \cdot M^{\prime}=\left[\begin{array}{c|c}
N . M & 0 \\
\hline 0 & 0
\end{array}\right], \quad \text { and }\left(N^{\prime} \cdot M^{\prime}\right)^{*}=\left[\begin{array}{c|c}
(N \cdot M)^{*} & 0 \\
\hline 0 & \mathrm{Id}_{m-n}
\end{array}\right] .
$$

Hence,

$$
(M . N)^{*}=\left(M^{\prime} . N^{\prime}\right)^{*}=\mathrm{Id}_{m}+M^{\prime} \cdot\left(N^{\prime} \cdot M^{\prime}\right)^{*} \cdot N^{\prime}=\mathrm{Id}_{m}+M \cdot(N \cdot M)^{*} \cdot N .
$$

Conversely,

$$
\begin{aligned}
\left(N^{\prime} \cdot M^{\prime}\right)^{*} & =\mathrm{Id}_{m}+N^{\prime} \cdot\left(M^{\prime} \cdot N^{\prime}\right)^{*} \cdot M^{\prime}=\mathrm{Id}_{m}+\left[\begin{array}{c|c}
N \cdot(M \cdot N)^{*} \cdot M & 0 \\
\hline 0 & 0
\end{array}\right] \\
& =\left[\begin{array}{c|c}
\mathrm{Id}_{n}+N \cdot(M \cdot N)^{*} \cdot M & 0 \\
\hline 0 & \mathrm{Id}_{m-n}
\end{array}\right] .
\end{aligned}
$$

Therefore, by identification of the first block, $(N \cdot M)^{*}=\mathrm{Id}_{n}+N .(M \cdot N)^{*} \cdot M$.

Proposition 6. Let $\mathbb{K}$ be a Conway semiring and let $m$ and $n$ be to nonnegative integers. The product 2 on matrices in $\mathbb{K}^{m+n}$ is associative and the unit is the usual identity matrix $\operatorname{ld}_{m+n}$.

Proof. To prove that $(M$ ¿ $N)$ ə $P=M$ ¿ $(N$ ə $P)$, we prove that the equality holds for the four 
blocks of these matrices.

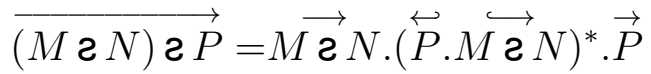

$$
\begin{aligned}
& =\vec{M} \cdot(\overleftrightarrow{N} \cdot \overleftrightarrow{M})^{*} \cdot \vec{N} \cdot\left(\overleftrightarrow{P} \cdot\left(\vec{N}+\overleftarrow{N} \cdot(\overleftrightarrow{M} \cdot \overleftrightarrow{N})^{*} \cdot \overleftrightarrow{M} \cdot \vec{N}\right)^{*} \cdot \vec{P}\right. \\
& =\vec{M} \cdot(\overleftrightarrow{N} \cdot \overleftrightarrow{M})^{*} \cdot \vec{N} \cdot\left((\overleftrightarrow{P} \cdot \vec{N})^{*} \cdot \overleftrightarrow{P} \cdot \overleftarrow{N} \cdot(\overleftrightarrow{M} \cdot \overleftrightarrow{N})^{*} \cdot \overleftrightarrow{M} \cdot \vec{N}\right)^{*} \cdot(\overleftrightarrow{P} \cdot \vec{N})^{*} \cdot \vec{P} \\
& =\vec{M} \cdot(\overleftrightarrow{N} \cdot \overleftrightarrow{M})^{*} \cdot\left(\vec{N} \cdot(\overleftrightarrow{P} \cdot \vec{N})^{*} \cdot \overleftrightarrow{P} \cdot \overleftarrow{N} \cdot(\overleftrightarrow{M} \cdot \overleftrightarrow{N})^{*} \cdot \overleftrightarrow{M}\right)^{*} \cdot \vec{N} \cdot(\overleftrightarrow{P} \cdot \vec{N})^{*} \cdot \vec{P} \\
& =\vec{M} \cdot(\overleftrightarrow{N} \cdot \overleftrightarrow{M})^{*} \cdot\left((\vec{N} \cdot \overleftrightarrow{P})^{*} \cdot \vec{N} \cdot \overleftrightarrow{P} \cdot \overleftarrow{N} \cdot \overleftrightarrow{M} \cdot(\overleftrightarrow{N} \cdot \overleftrightarrow{M})^{*}\right)^{*} \cdot \vec{N} \cdot(\overleftrightarrow{P} \cdot \vec{N})^{*} \cdot \vec{P} \\
& =\vec{M} \cdot\left(\left(\overleftrightarrow{N}+(\vec{N} \cdot \overleftrightarrow{P})^{*} \cdot \vec{N} \cdot \overleftrightarrow{P} \cdot \overleftarrow{N}\right) \cdot \overleftrightarrow{M}\right)^{*} \cdot \vec{N} \cdot(\overleftrightarrow{P} \cdot \vec{N})^{*} \cdot \vec{P}
\end{aligned}
$$

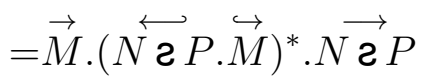

$$
\begin{aligned}
& =\overrightarrow{M \text { ¿ }(N \text { ¿ } P)} \text {. }
\end{aligned}
$$

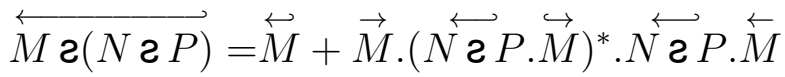

$$
\begin{aligned}
& =\overleftrightarrow{M}+\vec{M} \cdot\left[\left(\overleftrightarrow{N}+\vec{N} \cdot(\overleftrightarrow{P} \cdot \stackrel{\leftrightarrow}{N})^{*} \cdot \overleftrightarrow{P} \cdot \stackrel{\leftarrow}{N}\right) \cdot \overleftrightarrow{M}\right]^{*} \cdot\left(\overleftrightarrow{N}+\vec{N} \cdot(\overleftrightarrow{P} \cdot \overleftrightarrow{N})^{*} \cdot \overleftrightarrow{P} \cdot \overleftrightarrow{N}\right) \cdot \overleftrightarrow{M} \\
& =\overleftrightarrow{M}+\vec{M} \cdot(\overleftrightarrow{N} \cdot \overleftrightarrow{M})^{*} \cdot\left[\vec{N} \cdot(\overleftrightarrow{P} \cdot \vec{N})^{*} \cdot \overleftrightarrow{P} \cdot \overleftrightarrow{N} \cdot \overleftrightarrow{M} \cdot(\overleftrightarrow{N} \cdot \overleftrightarrow{M})^{*}\right]^{*} \\
& \cdot\left(\overleftrightarrow{N}+\vec{N} \cdot(\overleftrightarrow{P} \cdot \overleftrightarrow{N})^{*} \cdot \overleftrightarrow{P} \cdot \overleftarrow{N}\right) \cdot \overleftarrow{M} \\
& =\overleftrightarrow{M}+\vec{M} \cdot(\overleftrightarrow{N} \cdot \overleftrightarrow{M})^{*} \cdot\left[\vec{N} \cdot(\overleftrightarrow{P} \cdot \overleftrightarrow{N})^{*} \cdot \overleftrightarrow{P} \cdot \overleftrightarrow{N} \cdot \overleftrightarrow{M} \cdot(\overleftrightarrow{N} \cdot \overleftrightarrow{M})^{*}\right]^{*} \cdot \overleftrightarrow{N} \cdot \overleftrightarrow{M} \\
& +\vec{M} \cdot(\overleftrightarrow{N} \cdot \overleftrightarrow{M})^{*} \cdot \vec{N} \cdot\left[(\overleftrightarrow{P} \cdot \overleftrightarrow{N})^{*} \cdot \overleftrightarrow{P} \cdot \overleftrightarrow{N} \cdot \overleftrightarrow{M} \cdot(\overleftrightarrow{N} \cdot \overleftrightarrow{M})^{*} \cdot \vec{N}\right]^{*} \cdot(\overleftrightarrow{P} \cdot \stackrel{\leftrightarrow}{N})^{*} \cdot \overleftrightarrow{P} \cdot \overleftarrow{N} \cdot \overleftarrow{M} \\
& =\overleftrightarrow{M}+\vec{M} \cdot(\overleftrightarrow{N} \cdot \overleftrightarrow{M})^{*} \cdot \overleftrightarrow{N} \cdot \overleftarrow{M}+\vec{M} \cdot(\overleftrightarrow{N} \cdot \overleftrightarrow{M})^{*} \cdot \vec{N} \\
& {\left[(\overleftrightarrow{P} \cdot \overleftrightarrow{N})^{*} \cdot \overleftrightarrow{P} \cdot \overleftarrow{N} \cdot \overleftrightarrow{M} \cdot(\overleftrightarrow{N} \cdot \overleftrightarrow{M})^{*} \cdot \vec{N}\right]^{*} \cdot(\overleftrightarrow{P} \cdot \overleftrightarrow{N})^{*} \cdot \overleftrightarrow{P} \cdot \overleftarrow{N} \cdot \overleftrightarrow{M} \cdot(\overleftrightarrow{N} \cdot \overleftrightarrow{M})^{*} \cdot \overleftrightarrow{N} \cdot \overleftarrow{M}} \\
& +\vec{M} \cdot(\overleftrightarrow{N} \cdot \overleftrightarrow{M})^{*} \cdot \vec{N} \cdot\left[\overleftrightarrow{P} \cdot \stackrel{\leftrightarrow}{N}+\overleftrightarrow{\cdot P} \cdot \overleftrightarrow{N} \cdot \overleftrightarrow{M} \cdot(\overleftrightarrow{N} \cdot \overleftrightarrow{M})^{*} \cdot \vec{N}\right]^{*} \cdot \overleftrightarrow{P} \cdot \overleftrightarrow{N} \cdot \overleftarrow{M} \\
& =\overleftrightarrow{M \gtrsim N} \\
& +\overrightarrow{M \mathrm{e}} N \cdot\left(\overleftrightarrow{P} \cdot \overleftrightarrow{N}+\overleftrightarrow{P} \cdot \overleftarrow{N} \cdot \overleftrightarrow{M} \cdot(\overleftrightarrow{N} \cdot \overleftrightarrow{M})^{*} \cdot \vec{N}\right]^{*} \cdot \overleftrightarrow{P} \cdot \overleftarrow{N} \cdot \overleftrightarrow{M} \cdot(\overleftrightarrow{N} \cdot \overleftrightarrow{M})^{*} \cdot \overleftrightarrow{N} \cdot \overleftarrow{M} \\
& +\overrightarrow{M \imath} N \cdot\left[\overleftrightarrow{P} \cdot \overleftrightarrow{N}+\overleftrightarrow{P} \cdot \overleftarrow{N} \cdot \overleftrightarrow{M} \cdot(\overleftrightarrow{N} \cdot \overleftrightarrow{M})^{*} \cdot \vec{N}\right]^{*} \cdot \overleftrightarrow{P} \cdot \overleftarrow{N} \cdot \overleftarrow{M}
\end{aligned}
$$

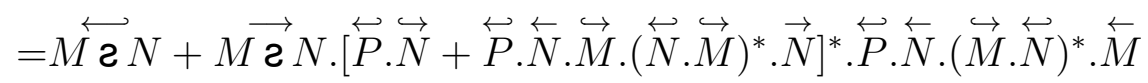

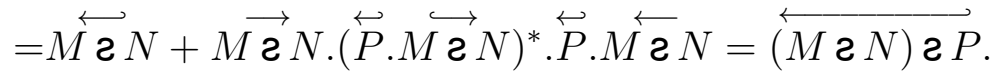

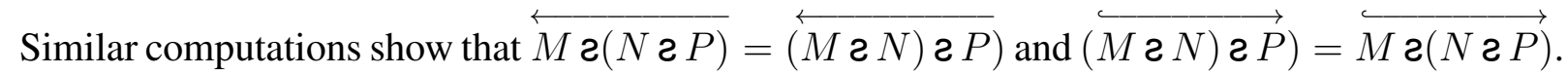




\subsection{Definition of two-way representations}

Definition 2. Let $\mathbb{K}$ be a Conway semiring and let $A$ be an alphabet. Let $m$ and $n$ be two nonnegative integers. A two-way representation over $A^{*}$ with dimension $m+n$ is a tuple $\rho=$ $(I, \mu, \diamond, T)$, where $I$ and $T$ are vectors in $\mathbb{K}^{m}, \mu$ is a morphism from $A^{*}$ into $\left(\mathbb{K}^{(m+n) \times(m+n)}, \mathbf{2}\right)$, and $\diamond$ is a matrix in $\mathbb{K}^{(m+n) \times(m+n)}$ such that $\vec{\diamond}=\operatorname{ld}_{m}$ and $\overleftarrow{\diamond}=0$

The series realized by $\rho$ is the series $|\rho|$ defined by

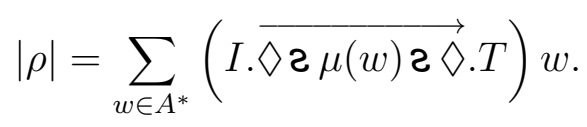

A series is two-way $\mathbb{K}$-recognizable if it can be realized by a two-way $\mathbb{K}$-representation.

In the sequel, we denote, for every word $w, \vec{\mu}(w)=\overrightarrow{\mu(w)}, \overleftrightarrow{\mu}(w)=\overleftrightarrow{\mu(w)}$, etc.

EXAMPLE. Let $\rho=(I, \diamond, \mu, T)$ be the two-way $\left(\mathbb{Q}_{+} \cup\{\infty\}\right)$-representation over $\{a\}^{*}$ with size $1+1$ defined by:

$$
I=[1], \quad T=[1], \quad \diamond=\left[\begin{array}{l|l}
1 & 0 \\
\hline 0 & 0
\end{array}\right], \quad \mu(a)=\left[\begin{array}{l|l}
1 / 2 & 1 / 2 \\
\hline 1 / 2 & 1 / 2
\end{array}\right]
$$

Hence, the weight of $a^{n}$ in $|\rho|$ is equal to $\vec{\mu}\left(a^{n}\right)$ for every $n$. We prove by induction that, for every $n, \vec{\mu}\left(a^{n}\right)=[1 /(n+1)]$ and $\overleftrightarrow{\mu}\left(a^{n}\right)=[n /(n+1)]$. It is true for $n=0$ and $n=1$; if it is true for $n-1$, then

$$
\begin{aligned}
\vec{\mu}\left(a^{n}\right) & =\vec{\mu}(a) \cdot\left(\overleftrightarrow{\mu}\left(a^{n-1}\right) \cdot \overleftrightarrow{\mu}(a)\right)^{*} \cdot \vec{\mu}\left(a^{n-1}\right)=\frac{1}{2}\left(\frac{n-1}{n} \cdot \frac{1}{2}\right)^{*} \frac{1}{n}=\frac{1}{2 n} \cdot \frac{1}{1-\frac{n-1}{2 n}}=\frac{1}{n+1} \\
\overleftrightarrow{\mu}\left(a^{n}\right) & =\overleftrightarrow{\mu}(a)+\vec{\mu}(a) \cdot\left(\overleftrightarrow{\mu}\left(a^{n-1}\right) \cdot \overleftrightarrow{\mu}(a)\right)^{*} \overleftrightarrow{\mu}\left(a^{n-1}\right) \cdot \overleftarrow{\mu}(a)=\frac{1}{2}+\frac{1}{2}\left(\frac{n-1}{n} \cdot \frac{1}{2}\right)^{*} \frac{n-1}{2 n} \\
& =\frac{1}{2}+\frac{n-1}{4 n} \cdot \frac{2 n}{n+1}=\frac{n}{n+1} .
\end{aligned}
$$

Therefore, $|\rho|=\sum_{k \geqslant 0} \frac{a^{k}}{k+1}$. This series is not in $\mathbb{Q R a t} a^{*}$; by [14], it is even not in the closure by the Hadamard product. Nevertheless, it belongs to $\mathbb{Q} H a d a^{*}$ :

$$
\begin{gathered}
a^{*}-\left(\frac{a}{2}\right)^{*} \cdot\left(\frac{a}{2}\right)^{*}=\sum_{k=0}^{\infty}\left(1-\frac{k+1}{2^{k}}\right) a^{k}, \\
\left(a^{*}-\left(\frac{a}{2}\right)^{*} \cdot\left(\frac{a}{2}\right)^{*}\right)^{\circledast}=\sum_{k=0}^{\infty} \frac{2^{k}}{k+1} a^{k}, \\
|\rho|=\left(a^{*}-\left(\frac{a}{2}\right)^{*} \cdot\left(\frac{a}{2}\right)^{*}\right)^{\circledast} \odot\left(\frac{a}{2}\right)^{*} .
\end{gathered}
$$

Notice that the two-way representation is over $\mathbb{Q}_{+} \cup\{\infty\}$, and this does not prove that $|\rho|$ is in $\left(\mathbb{Q}_{+} \cup\{\infty\}\right) \mathrm{Had} a^{*} ; \rho$ may even not be in $\left(\mathbb{Q}_{+} \cup\{\infty\}\right) \mathrm{RH} a^{*}$.

We propose an algorithm to compute $\langle|\rho|, w\rangle$ based on the following proposition. 
Proposition 7. Let $\mathbb{K}$ be a Conway semiring and let $\rho=(I, \mu, \diamond, T)$ be a two-way representation. Then for every word $w$ in $A^{*}$,

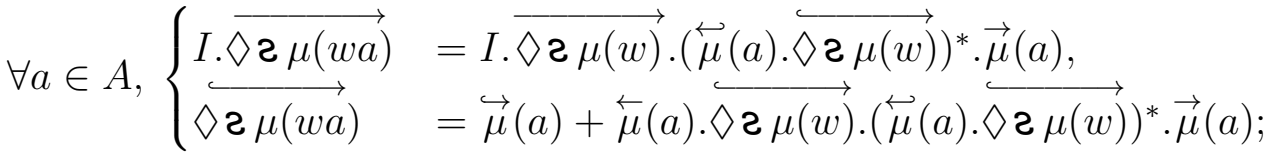

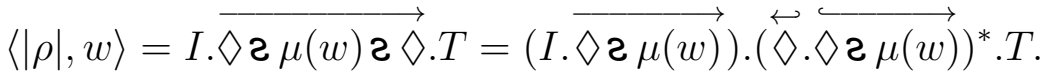

Proof. The two first equalities are straightforward from the definition of $\mu$ and the associativity of $ə$. The last one is also obvious since $\vec{\diamond}=\mathrm{Id}$.

Let $w=w_{1} \ldots w_{\ell}$ be a word of length $\ell$; to compute $\langle|\rho|, w\rangle$, we set $X_{0}=I$ and $Y_{0}=\overleftrightarrow{\diamond}$. Then, for every $i$ from 1 to $\ell$,

$$
X_{i}=X_{i-1} \cdot\left(\overleftrightarrow{\mu}\left(w_{i}\right) \cdot Y_{i-1}\right)^{*} \cdot \vec{\mu}\left(w_{i}\right), \quad Y_{i}=\overleftrightarrow{\mu}\left(w_{i}\right)+\overleftarrow{\mu}\left(w_{i}\right) \cdot Y_{i-1} \cdot\left(\overleftrightarrow{\mu}\left(w_{i}\right) \cdot Y_{i-1}\right)^{*} \cdot \vec{\mu}\left(w_{i}\right)
$$

Finally, $\langle|\rho|, w\rangle=X_{\ell} \cdot\left(\overleftrightarrow{\diamond} \cdot Y_{\ell}\right)^{*} . T$

The complexity of this computation depends on the complexity of the operations in the semiring $\mathbb{K}$; usually, the addition is less expensive than the multiplication; moreover, the star of a matrix of size $n$ can be computed with $O\left(n^{3}\right)$ multiplications (for instance with the McNaughtonYamada algorithm [10]). Notice that we consider the naive algorithm for the multiplication of matrices: for every $n, m, r$, the multiplication of $M$ in $\mathbb{K}^{m \times r}$ and $N$ in $\mathbb{K}^{r \times n}$ can be performed with $O(m n r)$ multiplications. We evaluate the complexity of each step of the algorithm. Assume that $\rho$ is a representation with size $m+n$; for every $i, X_{i}$ is a vector of size $m$ and $Y_{i}$ is a matrix of size $n \times m$.

- the product $\overleftrightarrow{\mu}\left(w_{i}\right) \cdot Y_{i-1}$ requires $O\left(m^{2} n\right)$ multiplications;

- the $\operatorname{star}\left(\overleftrightarrow{\mu}\left(w_{i}\right) \cdot Y_{i-1}\right)^{*}$ requires $O\left(m^{3}\right)$ multiplications;

- the product $X_{i-1} \cdot\left(\overleftrightarrow{\mu}\left(w_{i}\right) \cdot Y_{i-1}\right)^{*}$ requires $O\left(m^{2}\right)$ multiplications;

- the product $X_{i-1} \cdot\left(\overleftarrow{\mu}\left(w_{i}\right) \cdot Y_{i-1}\right)^{*} \cdot \vec{\mu}\left(w_{i}\right)$ requires $O\left(m^{2}\right)$ multiplications;

- the product $Y_{i-1} \cdot\left(\overleftrightarrow{\mu}\left(w_{i}\right) \cdot Y_{i-1}\right)^{*}$ requires $O\left(m^{2} n\right)$ multiplications;

- the product $Y_{i-1} \cdot\left(\overleftrightarrow{\mu}\left(w_{i}\right) \cdot Y_{i-1}\right)^{*} \cdot \vec{\mu}\left(w_{i}\right)$ requires $O\left(m^{2} n\right)$ multiplications;

- the product $\overleftarrow{\mu}\left(w_{i}\right) \cdot Y_{i-1} \cdot\left(\overleftrightarrow{\mu}\left(w_{i}\right) \cdot Y_{i-1}\right)^{*} \cdot \vec{\mu}\left(w_{i}\right)$ requires $O\left(m^{2} n\right)$ multiplications

Thus, at each step, the cost of matrix multiplications is $O\left(m^{2} n\right)$ and the cost of the star of the matrix is $O\left(\mathrm{~m}^{3}\right)$; notice that if $n$ is negligible w.r.t. $m$, thanks to the identity $(M . N)^{*}=\mathrm{Id}+$ $M .(N . M)^{*} . N$, this cost can be converted into $O\left(n^{3}\right)$ (with $O\left(m n^{2}\right)$ auxilliary multiplications). The cost of the final computation is similar, and finally:

Proposition 8. Let $\rho$ be a two-way representation of size $m+n$. The coefficient of a word of length $k$ in $|\rho|$ can be computed with $O\left(k\left(m^{2} n+\min \left(m^{3}+n^{3}\right)\right)\right)$ multiplications. 
Classes of two-way $\mathbb{K}$-representations. We shall study the expressiveness of some subclasses of two-way $\mathbb{K}$-representations. Let $\rho=(I, \mu, \diamond, T)$ be a two-way $\mathbb{K}$-representation of size $m+n$.

- $\rho$ is a sweeping $\mathbb{K}$-representation if for every letter $a, \overleftrightarrow{\mu}(a)=0$ and $\overleftrightarrow{\mu}(a)=0$.

- $\rho$ is a rotating $\mathbb{K}$-representation if it is sweeping and, for every letter $a, \overleftarrow{\mu}(a)=\mathrm{Id}$

- If $n=0, \rho$ is one-way. In this case, $\diamond=\mathrm{Id}$ can be ignored and the representation is a linear $\mathbb{K}$-representation, as defined in [9]. By the Kleene-Schützenberger Theorem [15], a series over $A^{*}$ can be realized by a one-way $\mathbb{K}$-representation if and only if it is in $\mathbb{K}$ Rat $A^{*}$.

\subsection{Closure properties}

Like in the case of linear (one-way) representations, the set of series realized by two-way representations is closed by a number of operations.

Proposition 9. The set of series realized by two-way (resp. sweeping, resp. rotating) $\mathbb{K}$ representations is closed under the entrywise operations.

Proof. Let $\rho_{i}=\left(I_{i}, \mu_{i}, \diamond_{i}, T_{i}\right)$ be a representation of size $m_{i}+n_{i}$, for $i$ in $\{1,2\}$.

- SuM. Let $\rho_{3}=\left(I_{3}, \mu_{3}, \diamond_{3}, T_{3}\right)$ be the representation of size $\left(m_{1}+m 2\right)+\left(n_{1}+n_{2}\right)$ defined by

$$
\begin{aligned}
& I_{3}=\left[\begin{array}{l|l}
I & J
\end{array}\right], \quad T_{3}=\left[\begin{array}{c}
T_{1} \\
\hline T_{2}
\end{array}\right], \quad \forall a \in A,
\end{aligned}
$$

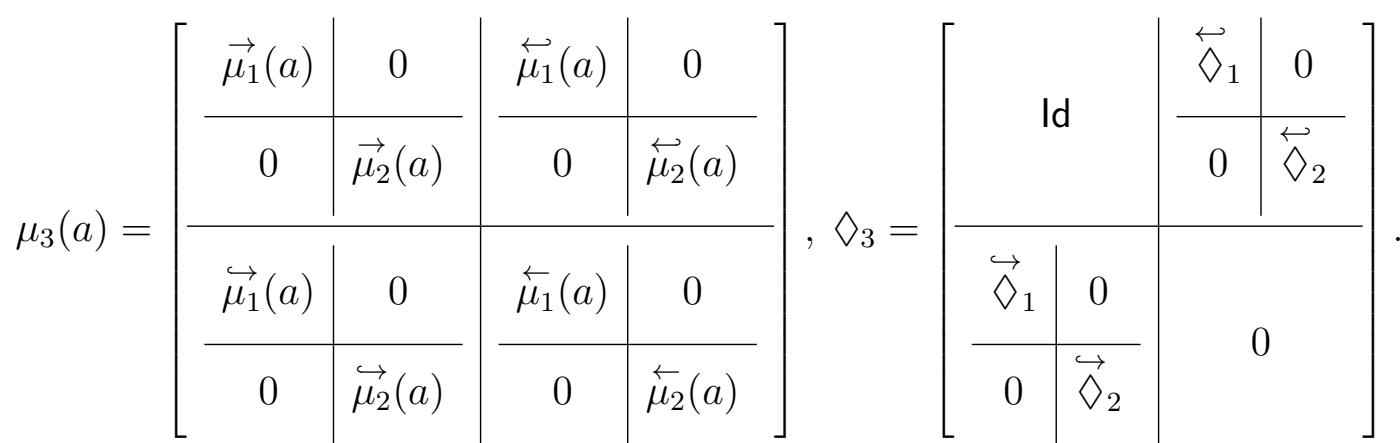

By induction on the length of words, it immediatly comes that, for every non empty word $w$,

$$
\mu_{3}(w)=\left[\begin{array}{c|c|c|c}
\overrightarrow{\mu_{1}}(w) & 0 & \overleftarrow{\mu_{1}}(w) & 0 \\
\hline 0 & \overrightarrow{\mu_{2}}(w) & 0 & \overleftarrow{\mu_{2}}(w) \\
\hline \overleftrightarrow{\mu_{1}}(w) & 0 & \overleftarrow{\mu_{1}}(w) & 0 \\
\hline 0 & \overleftrightarrow{\mu_{2}}(w) & 0 & \overleftarrow{\mu_{2}}(w)
\end{array}\right]
$$


Hence, for every word $w$,

$$
\begin{aligned}
& \left.I_{3} \cdot \overrightarrow{\nabla_{3}} \boldsymbol{2} \mu_{3}(w)=I_{3} \cdot\left(\overleftrightarrow{\mu_{3}}(w) \cdot \overleftrightarrow{\nabla_{3}}\right)^{*} \cdot \overrightarrow{\mu_{3}}(w)\right) \\
& \left.\left.=\left[I_{1} \cdot\left(\overleftrightarrow{\mu_{1}}(w) \cdot \overleftrightarrow{\nabla_{1}}\right)^{*} \cdot \overrightarrow{\mu_{1}}(w)\right) \mid I_{2} \cdot\left(\overleftrightarrow{\mu_{2}}(w) \cdot \overleftrightarrow{\diamond_{2}}\right)^{*} \cdot \overrightarrow{\mu_{2}}(w)\right)\right] \\
& =\left[I_{1} \cdot \overrightarrow{\nabla_{1} \curvearrowright \mu_{1}(w)} \mid I_{2} \cdot \overrightarrow{\nabla_{2} \curvearrowright \mu_{2}(w)}\right] \text {, }
\end{aligned}
$$

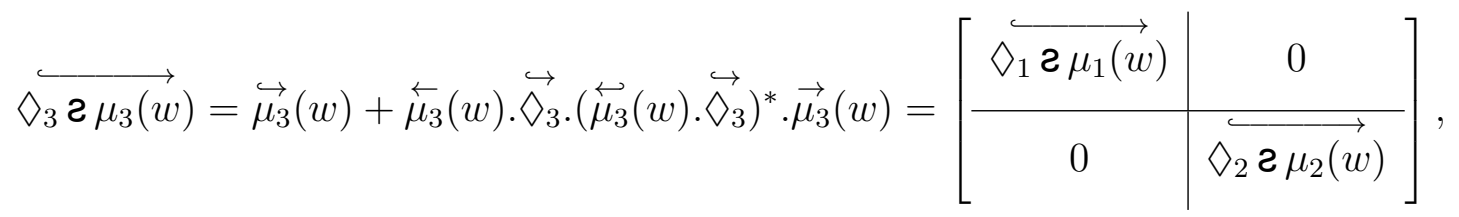

and finally

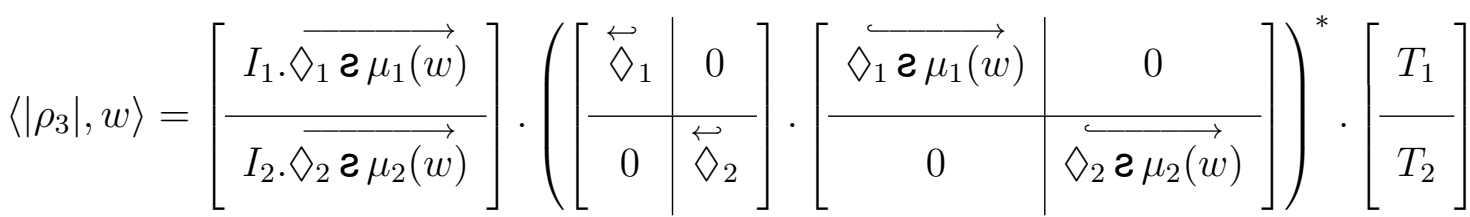

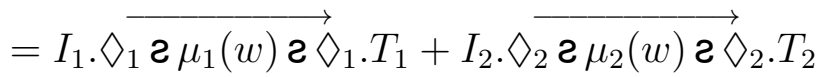

$$
\begin{aligned}
& =\left\langle\left|\rho_{1}\right|, w\right\rangle+\left\langle\left|\rho_{2}\right|, w\right\rangle=\left\langle\left|\rho_{1}\right|+\left|\rho_{2}\right|, w\right\rangle \text {. }
\end{aligned}
$$

- HADAMARD PRODUCT. Let $\rho_{4}=\left(I_{4}, \mu_{4}, \diamond_{4}, T_{4}\right)$ be the representation of size $\left(m_{1}+\right.$ $\left.m_{2}\right)+\left(n_{1}+1+n_{2}\right)$ defined by:

$$
\begin{aligned}
& I_{4}=\left[\begin{array}{lll}
I_{1} & 0
\end{array}\right], \quad T_{4}=\left[\begin{array}{c}
0 \\
\hline T_{2}
\end{array}\right], \forall a \in A
\end{aligned}
$$

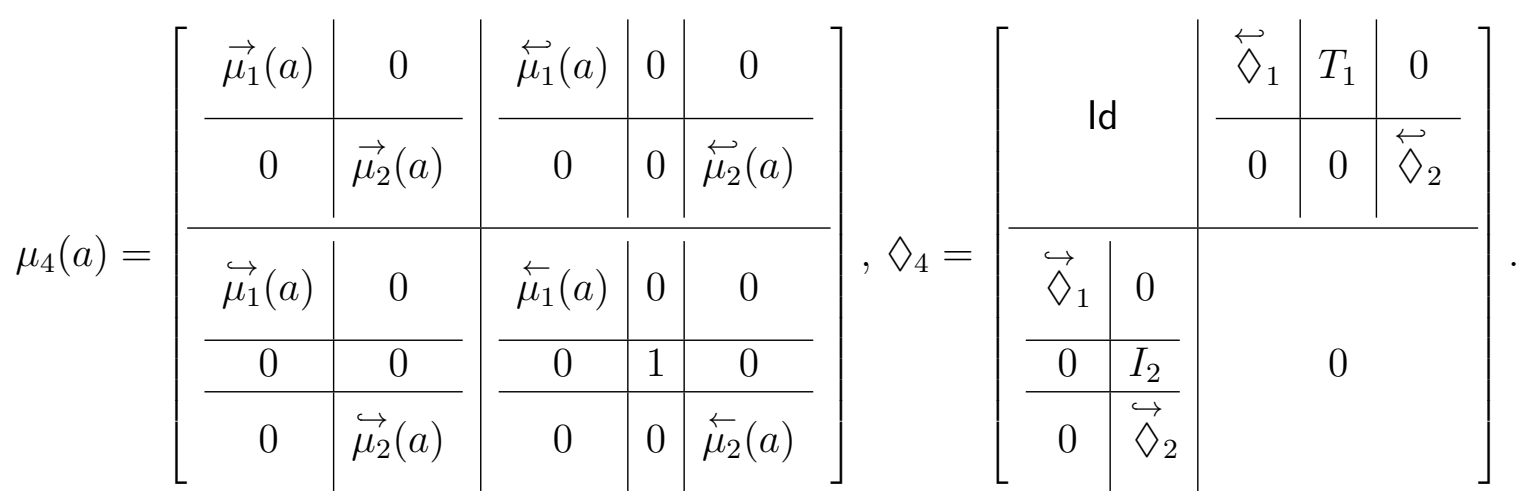


We prove by induction that, for every word $w$,

$$
\begin{aligned}
& I_{4} \cdot \overrightarrow{\nabla_{4} \approx \mu_{4}(w)}=\left[I_{1} \cdot \overrightarrow{\nabla_{1} \approx \mu_{1}(w)} \mid 0\right] \text {, }
\end{aligned}
$$

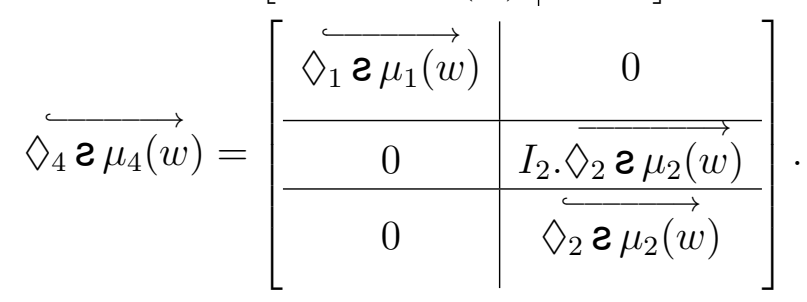

It is true if $w$ if the empty word, and, if it is true for $w$, for every letter $a$ :

$$
\begin{aligned}
& \left(\overleftrightarrow{\mu_{4}}(a) \cdot \diamond_{4} \approx \mu_{4}(w)\right)^{*} \cdot \vec{\mu}_{4}(a)
\end{aligned}
$$

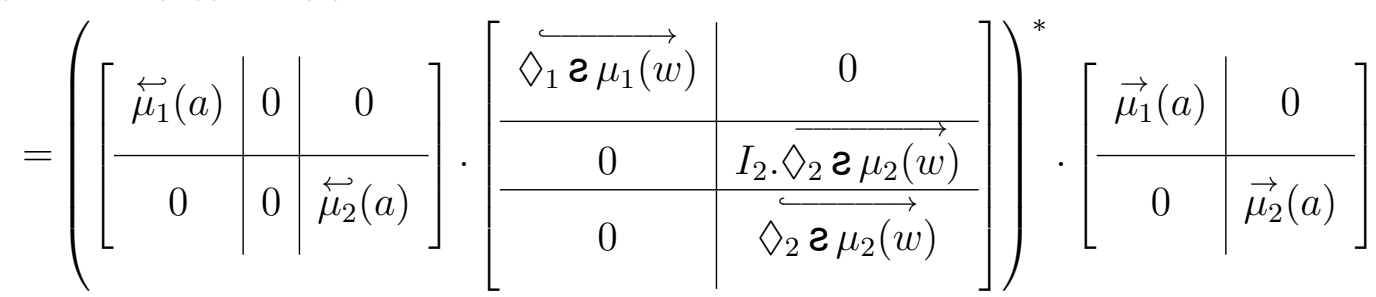

$$
\begin{aligned}
& =\left[\begin{array}{c|c|c}
\overleftrightarrow{\mu_{1}}(a) \cdot \overleftrightarrow{\diamond_{1} 2 \mu_{1}(w)} & 0 \\
\hline 0 & \overleftrightarrow{\mu_{1}}(a) \cdot \diamond_{2} 2 \mu_{2}(w)
\end{array}\right]^{*} \cdot\left[\begin{array}{cc}
\overrightarrow{\mu_{1}}(a) & 0 \\
\hline 0 & \overrightarrow{\mu_{2}}(a)
\end{array}\right] \\
& =\left[\begin{array}{c|c}
\left(\overleftrightarrow{\mu_{1}}(a) \cdot \overleftrightarrow{\diamond_{1} 2 \mu_{1}(w)}\right)^{*} \cdot \overrightarrow{\mu_{1}}(a) & 0 \\
\hline 0 & \left(\overleftrightarrow{\mu_{1}}(a) \cdot \diamond_{2} 2 \mu_{2}(w)\right)^{*} \cdot \overrightarrow{\mu_{2}}(a) .
\end{array}\right]
\end{aligned}
$$

$$
\begin{aligned}
& I_{4} \cdot \diamond_{4} \text { ¿ } \mu_{4}(w a)
\end{aligned}
$$

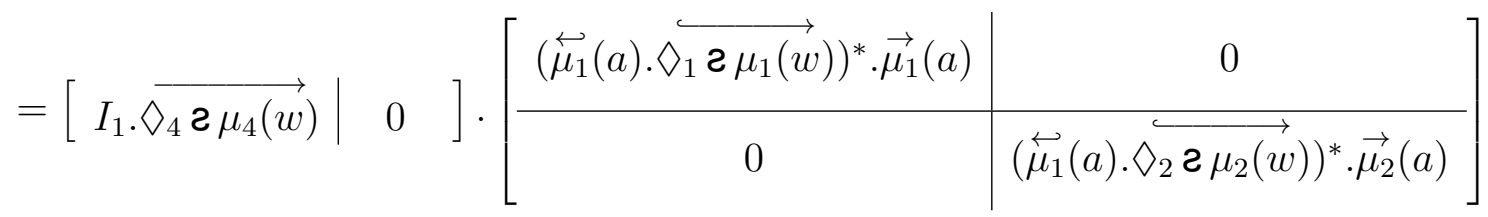

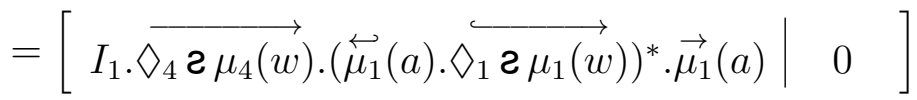

$$
\begin{aligned}
& =\left[\begin{array}{l|l}
I_{1} . \diamond_{1} 2 \mu_{1}(w a) & 0
\end{array}\right] \text {. }
\end{aligned}
$$


$\diamond_{4} \curvearrowright \mu_{4}(w a)$

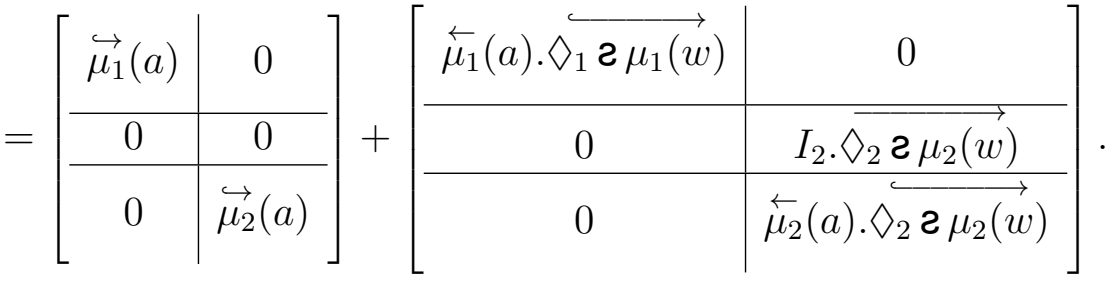

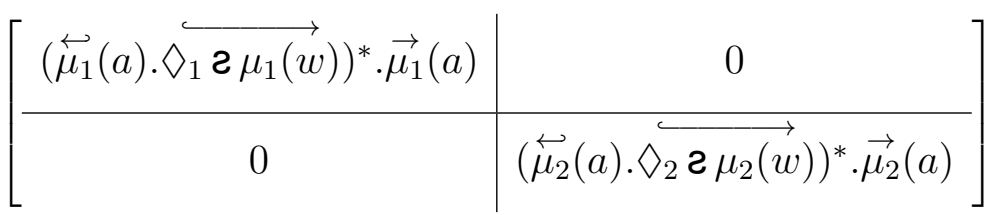

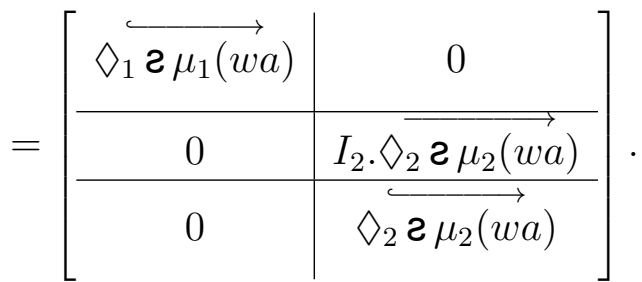

We then compute $\left(\overleftrightarrow{\nabla_{4}} \cdot \triangleleft_{4} 2 \mu_{4}(w)\right)^{*}$ :

$$
\begin{aligned}
& \left(\left[\begin{array}{c|c|c}
\overleftrightarrow{\diamond}_{1} & T_{1} & 0 \\
\hline 0 & 0 & \overleftrightarrow{\diamond}_{2}
\end{array}\right] \cdot\left[\begin{array}{c|c}
\stackrel{\diamond_{1} 2 \mu_{1}(w)}{\longrightarrow} & 0 \\
\hline 0 & I_{2} \cdot \overrightarrow{\diamond_{2} 2 \mu_{2}(w)} \\
\hline 0 & \diamond_{2} 2 \mu_{2}(w)
\end{array}\right]\right)^{*}
\end{aligned}
$$

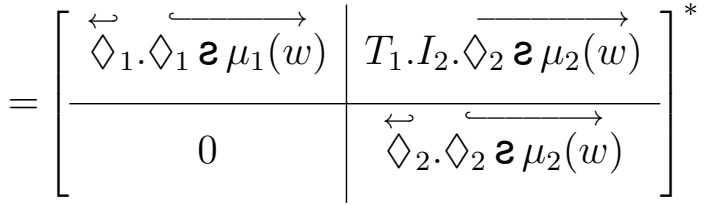

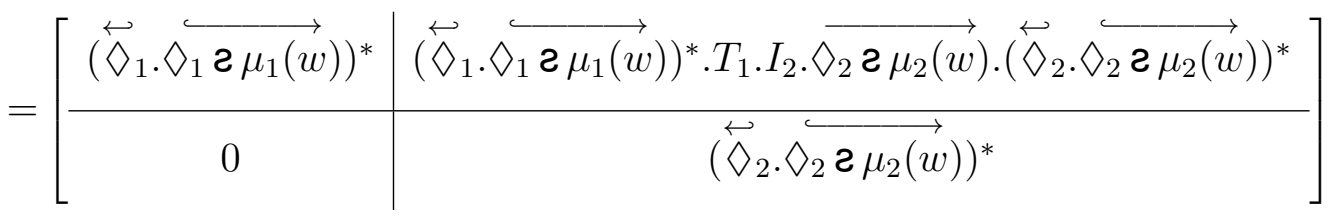

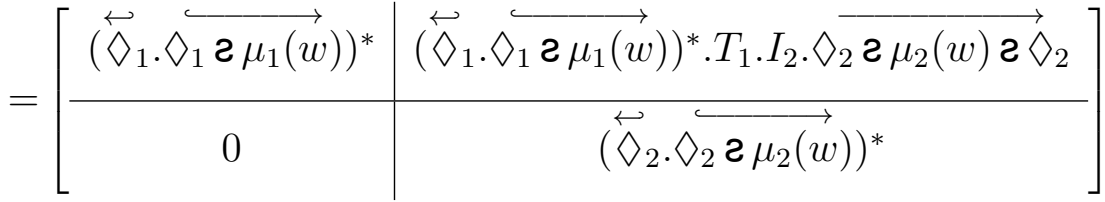


Finally, the coefficient of $w$ in $\left|\rho_{4}\right|$ is

$$
\begin{aligned}
& \left(I_{4} \cdot \overrightarrow{\nabla_{4} 2 \mu_{4}(w)}\right) \cdot\left(\overleftrightarrow{\nabla_{4}} \cdot \diamond_{4} 2 \mu_{4}(w a)\right)^{*} \cdot T_{4}
\end{aligned}
$$

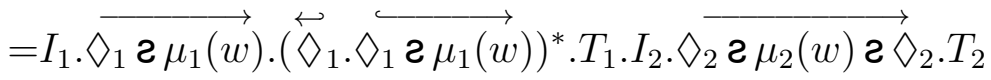

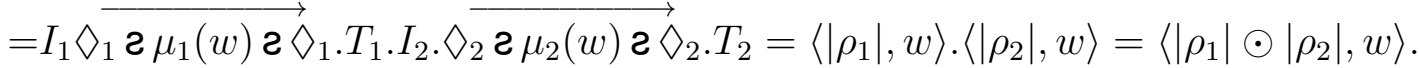

- Hadamard iteration. Let $\rho=(I, \mu, \diamond, T)$ be a two-way representation and let $\rho_{5}$ be the two-way representation defined by

$$
\begin{aligned}
& I_{5}=\left[\begin{array}{l|l}
1 & I
\end{array}\right], T_{5}=\left[\begin{array}{c}
1 \\
T
\end{array}\right] \text {, } \\
& \forall a \in A, \mu_{5}(a)=\left[\begin{array}{c|c|c|c}
1 & 0 & 0 & 0 \\
\hline 0 & \vec{\mu}(a) & \overleftarrow{\mu}(a) & 0 \\
\hline 0 & \overleftrightarrow{\mu}(a) & \overleftarrow{\mu}(a) & 0 \\
\hline 0 & 0 & 0 & 1
\end{array}\right], \quad \nabla_{5}=\left[\begin{array}{c|c|c}
\text { Id } & \overleftarrow{\Delta} & T \\
\hline 0 & \overleftrightarrow{\diamond} & \multicolumn{2}{|c}{0} \\
\hline 0 & I
\end{array}\right]
\end{aligned}
$$

We prove by induction that, for every word $w$,

$$
\begin{aligned}
& I_{5} \cdot \overrightarrow{\nabla_{5} \curvearrowright \mu_{5}(w)}=\left[1 \mid I . \overrightarrow{\nabla \_\mu(w)}\right],
\end{aligned}
$$

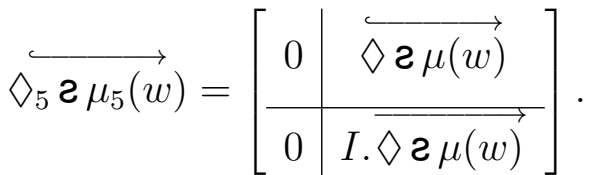

It is true if $w$ if the empty word, and, if it is true for $w$, for every letter $a$ :

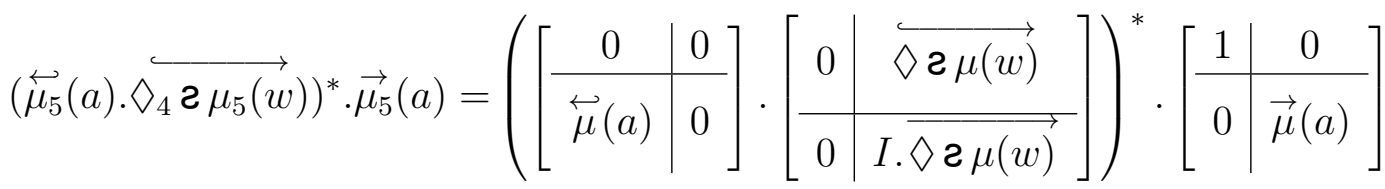

$$
\begin{aligned}
& =\left[\begin{array}{c|c}
0 & 0 \\
\hline 0 & \overleftrightarrow{\mu}(a) \cdot \stackrel{\diamond \boldsymbol{\iota} \mu(w)}{\longrightarrow}
\end{array}\right]^{*} \cdot\left[\begin{array}{c|c}
1 & 0 \\
\hline 0 & \vec{\mu}(a)
\end{array}\right] \\
& =\left[\begin{array}{c|c}
1 & 0 \\
\hline 0 & (\overleftrightarrow{\mu}(a) \cdot \circlearrowleft 2 \mu(w))^{*} \cdot \vec{\mu}(a)
\end{array}\right] \text {. }
\end{aligned}
$$




$$
\begin{aligned}
& I_{5} \cdot \nabla_{5} \curvearrowright \mu_{5}(w a) \\
& =[1 \mid I . \overrightarrow{\nabla \Sigma \mu(w)}] \cdot\left[\begin{array}{c|c}
1 & 0 \\
\hline 0 & (\overleftrightarrow{\mu}(a) \cdot \triangleleft 2 \mu(w))^{*} \cdot \vec{\mu}(a)
\end{array}\right]
\end{aligned}
$$

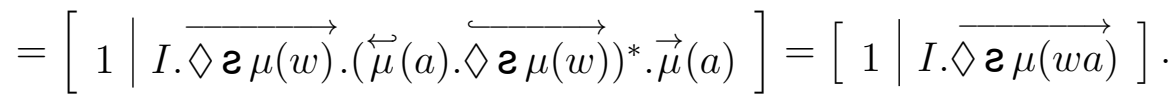

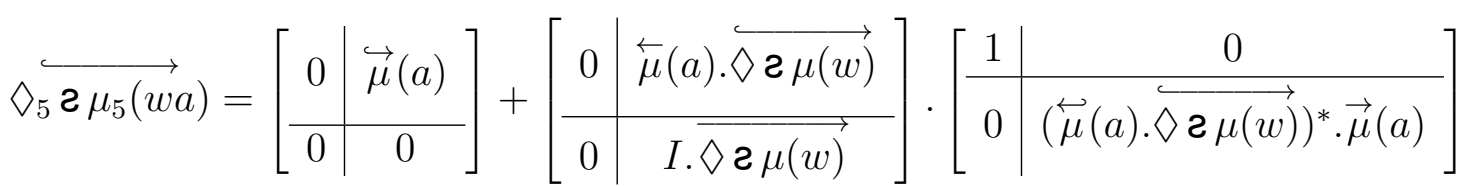

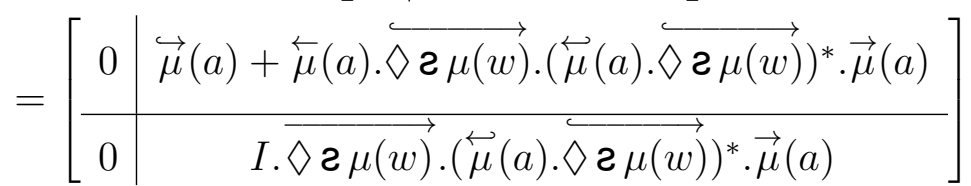

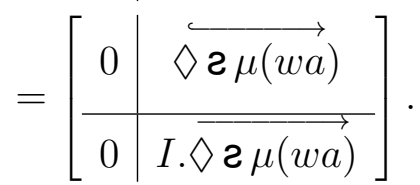

We then compute $\left(\overleftrightarrow{\nabla_{5}} \cdot \triangleleft_{5} \text { 己 } \mu_{5}(w)\right)^{*}$ :

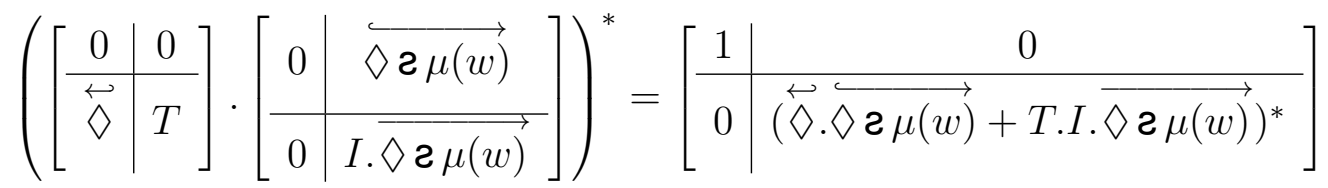

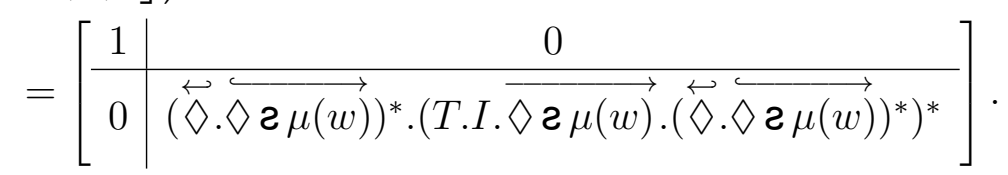

Finally, the coefficient of $w$ in $\left|\rho_{5}\right|$ is

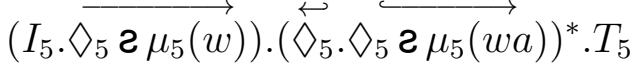

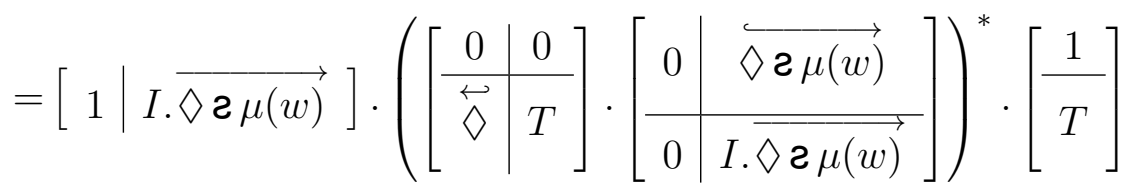

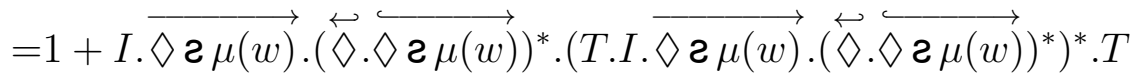

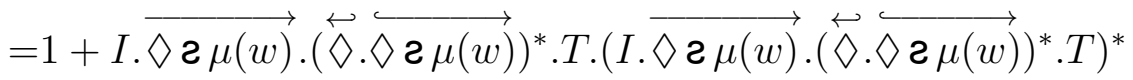

$$
\begin{aligned}
& =1+\langle|\rho|, w\rangle \cdot\langle|\rho|, w\rangle^{*}=\langle|\rho|, w\rangle^{*} \text {. }
\end{aligned}
$$

Notice that these constructions preserve the sweeping and the rotating properties. 
Proposition 10. The set of series realized by two-way (resp. sweeping,) $\mathbb{K}$-representations is closed under mirror.

Proof. Let $\rho=(I, \mu, \diamond, T)$ be a two-way representation of size $m+n$ and let $\rho_{6}$ be the two-way representation of size $(m+2)+n$ defined by

$$
\begin{aligned}
& I_{6}=\left[\begin{array}{l|ll}
1 & 0 & 0
\end{array}\right], T_{6}=\left[\begin{array}{c}
0 \\
0 \\
\hline 1
\end{array}\right],
\end{aligned}
$$

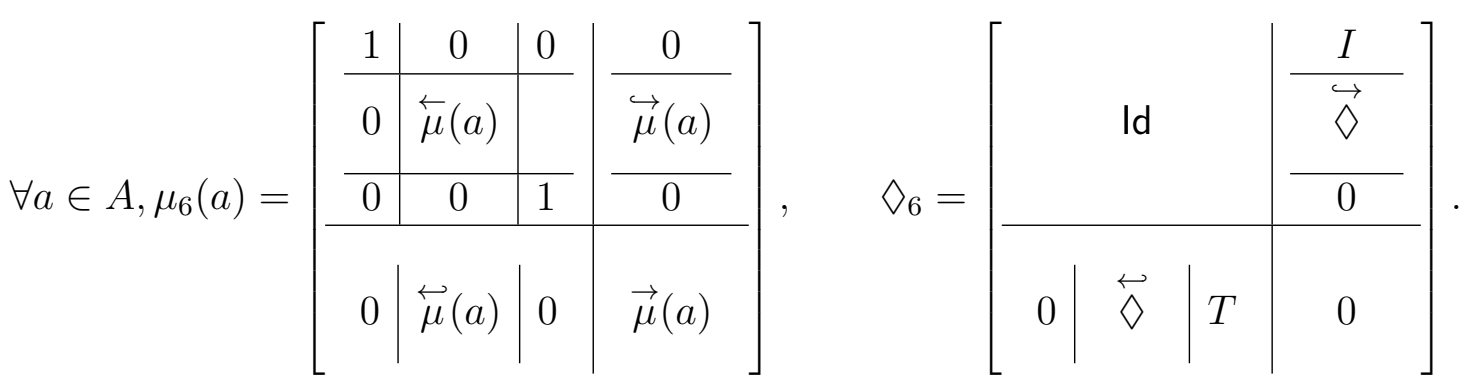

We prove by induction that, for every word $w$,

$$
\begin{aligned}
& I_{6} \cdot \overrightarrow{\nabla_{6}} \text { 己 } \mu_{6}(w)=I_{6}
\end{aligned}
$$

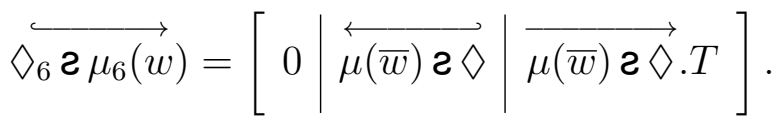

It is true if $w$ if the empty word, and, if it is true for $w$, for every letter $a$ :

$$
\begin{aligned}
& \left(\overleftrightarrow{\mu_{6}}(a) \cdot \overleftrightarrow{\searrow_{6} 2 \mu_{6}(w)}\right)^{*} \cdot \overrightarrow{\mu_{6}}(a)
\end{aligned}
$$

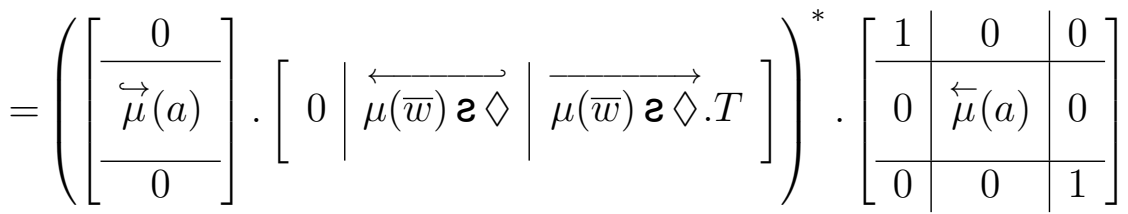

$$
\begin{aligned}
& =\left[\begin{array}{c|c|c}
0 & 0 & 0 \\
\hline 0 & \overleftrightarrow{\mu}(a) \cdot \overleftrightarrow{\mu(\bar{w}) \boldsymbol{\diamond}} & \overleftrightarrow{\mu}(a) \cdot \overrightarrow{\mu(\bar{w}) \boldsymbol{\diamond} \cdot T} \\
\hline 0 & 0 & 0
\end{array}\right]^{*} \cdot\left[\begin{array}{c|c|c}
1 & 0 & 0 \\
\hline 0 & \overleftarrow{\mu}(a) & 0 \\
\hline 0 & 0 & 1
\end{array}\right]
\end{aligned}
$$

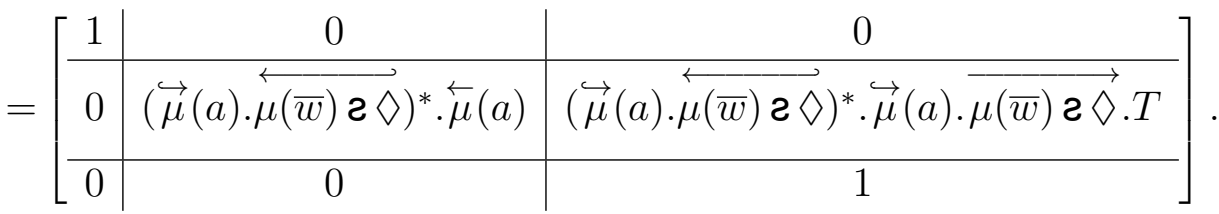

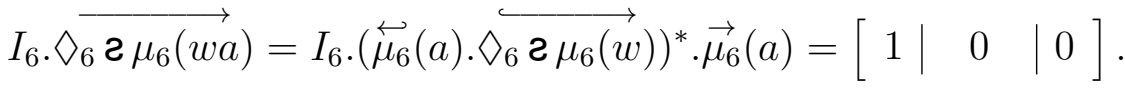




$$
\begin{aligned}
& \diamond_{6} 2 \mu_{6}(w a)
\end{aligned}
$$

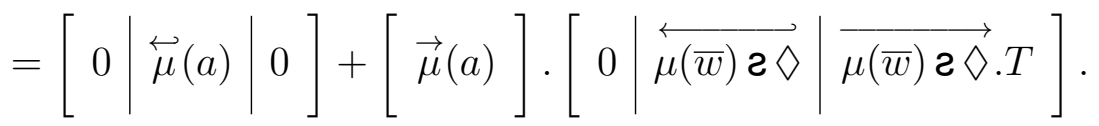

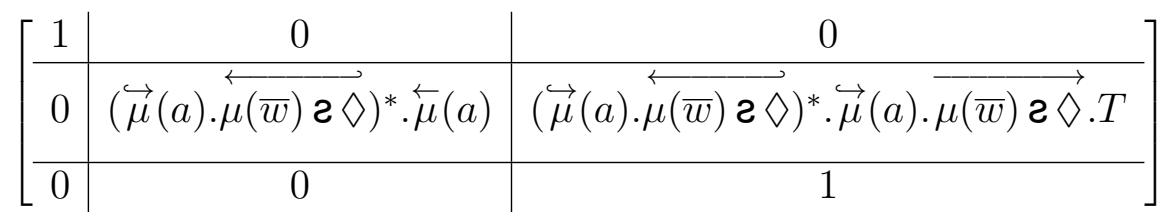

$$
\begin{aligned}
& =[0|\overleftrightarrow{\mu}(a)| 0]+[0|\vec{\mu}(a) \cdot \overleftrightarrow{\mu(\bar{w}) \boldsymbol{\iota} \diamond}| \vec{\mu}(a) \cdot \overrightarrow{\mu(\bar{w}) \boldsymbol{乙} \diamond \cdot T}]
\end{aligned}
$$

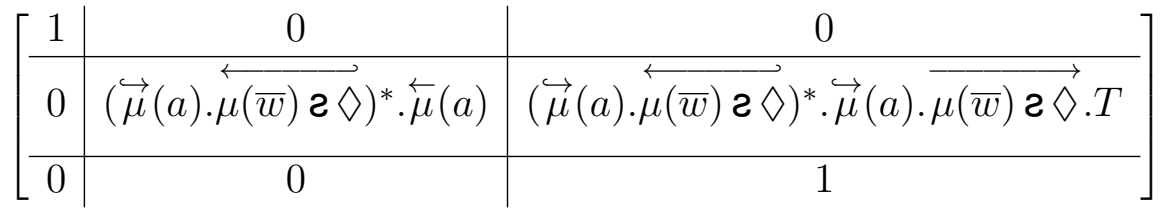

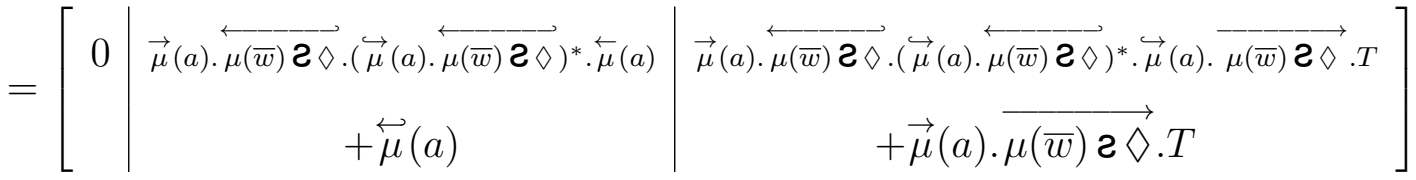

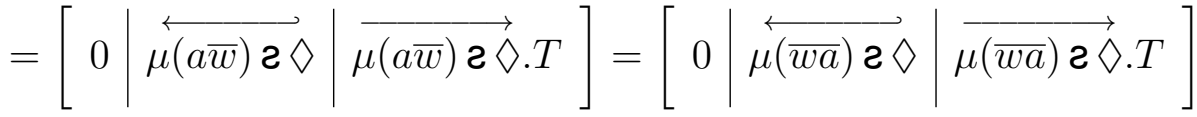

Finally, the coefficient of $w$ in $\left|\rho_{6}\right|$ is

$$
\begin{aligned}
& \left(I_{6} \cdot \overrightarrow{\nabla_{6}} \text { 己 } \mu_{5}(w)\right) \cdot\left(\overleftrightarrow{\nabla_{6}} \cdot \diamond_{6} \text { 己 } \mu_{6}(w a)\right)^{*} \cdot T_{6} \\
& =\left[\begin{array}{l|ll}
1 & 0 & 0
\end{array}\right] \cdot\left(\left[\begin{array}{c}
\frac{I}{\overleftrightarrow{\diamond}} \\
\hline 0
\end{array}\right] \cdot[0|\overleftrightarrow{\mu(\bar{w}) \boldsymbol{\iota} \diamond}| \overrightarrow{\mu(\bar{w}) \boldsymbol{\partial} \diamond \cdot T}]\right)^{*} \cdot\left[\begin{array}{c}
\frac{0}{0} \\
\hline 1
\end{array}\right]
\end{aligned}
$$

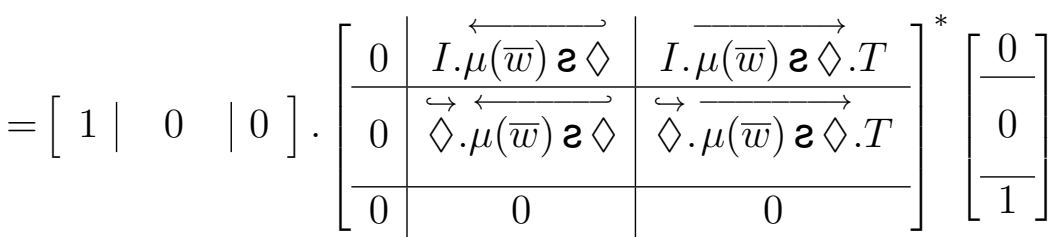

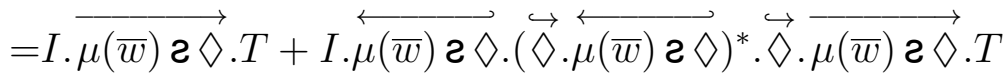

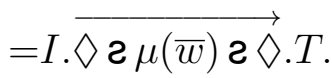

This construction preserves the sweeping property.

Proposition 11. Let $\mathbb{K}$ be a Conway semiring and $A$ an alphabet.

Every series in $\mathbb{K} \mathrm{Had} A^{*}$ can be realized by a rotating $\mathbb{K}$-representation.

Every series in $\mathbb{K}$ MirHad $A^{*}$ can be realized by a sweeping $\mathbb{K}$-representation. 
Proof. Every series in $\mathbb{K}$ Rat $A^{*}$ can be realized by a one-way $\mathbb{K}$-representation, and the set of series realizable by rotating $\mathbb{K}$-representations is closed by the entrywise operations. Likewise, the set of series realizable by sweeping $\mathbb{K}$-representations is closed by mirror.

Proposition 12. Let $\mathbb{K}$ be a Conway semiring, let $A$ be an alphabet, and let $\rho=(I, \mu, \diamond, T)$ be a two-way $\mathbb{K}$-representation over $A^{*}$. For every word $u$, the left quotient of $\rho$ by $u$,

$$
u^{-1}|\rho|=\sum_{v \in A^{*}}\langle|\rho|, u v\rangle v
$$

is realized by the two-way representation $\left(I^{\prime}, \mu, \diamond^{\prime}, T\right)$, with

$$
I^{\prime}=I . \overrightarrow{\nabla \mathbf{z} \mu(u)}, \quad \diamond^{\prime}=\left[\begin{array}{c|c}
\mathrm{Id} & \overleftrightarrow{\nabla \mathbf{2} \mu(u)} \\
\hline \overleftrightarrow{\diamond} & 0
\end{array}\right]
$$

Proof. First, notice that $\diamond^{\prime}$ acts as $\diamond$ when applied to the right:

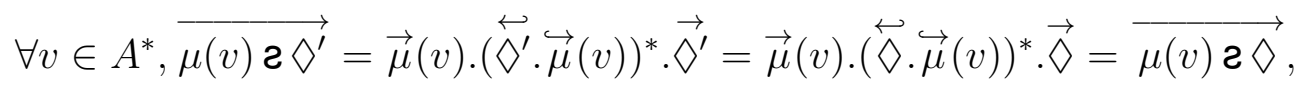

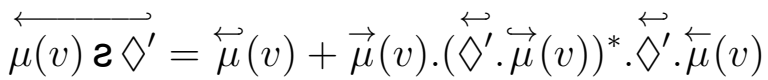

$$
\begin{aligned}
& =\overleftrightarrow{\mu}(v)+\vec{\mu}(v) \cdot(\overleftrightarrow{\Delta} \cdot \overleftrightarrow{\mu}(v))^{*} \cdot \overleftrightarrow{\diamond} \cdot \overleftarrow{\mu}(v)=\overleftrightarrow{\mu(v) 己 \diamond}
\end{aligned}
$$

Then,

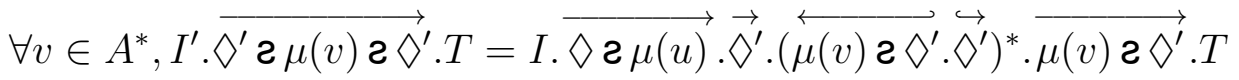

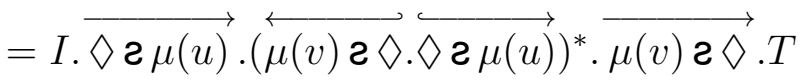

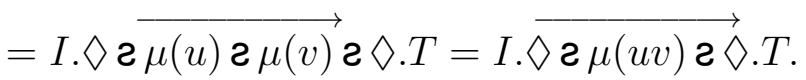

We shall see further that two-way recognizable series are not closed under Cauchy product and Kleene star.

\section{Two-way-recognizable series as fixed points}

In the case of one-way $\mathbb{K}$-automata, if $M$ is the transition matrix of an automaton with final vector $T$, and $X$ is the vector with entry in $\mathbb{K}\left\langle\left\langle A^{*}\right\rangle\right\rangle$ such that $X_{p}$ is the series realized if $p$ is the initial state (with weight 1 ), $X$ fulfills the following fixed-point equation:

$$
X=T+M \cdot X \text {. }
$$


If each entry of $M$ is a linear combination of letters, $S=M^{*} . T$ is the unique solution of the equation, and thus the series realized by the automaton is $I . M^{*} . T$, where $I$ is the initial vector. This is the foundation of algorithms that convert one-way automata to rational expressions.

We set a similar equation for two-way representations. We consider the matrix algebras respectively induced by the Cauchy and the Hadamard products. For every $m, h, n$ in $\mathbb{N}$, for every $M$ in $\mathbb{K}\left\langle\left\langle A^{*}\right\rangle\right\rangle^{m \times h}$ and every $N$ in $\mathbb{K}\left\langle\left\langle A^{*}\right\rangle\right\rangle^{h \times n}$, for every $(i, j)$ in $[1 ; m] \times[1 ; n]$,

$$
(M \cdot N)_{i, j}=\sum_{k \in[1 ; h]} M_{i, k} \cdot M_{k, j}, \quad(M \odot N)_{i, j}=\sum_{k \in[1 ; h]} M_{i, k} \odot M_{k, j} .
$$

Notice that $M \odot N$ is not the Hadamard (entrywise) product of matrices.

These matrix products admit unit elements : $\operatorname{ld}_{n}$ is the diagonal matrix of size $n$ with entries 1 and $A_{n}^{*}$ is the diagonal matrix with entries $1_{\odot}$ (the dimension may be omitted).

If $\mathbb{K}$ is a Conway semiring, so is the semiring $\left(\mathbb{K}\left\langle\left\langle A^{*}\right\rangle\right\rangle,+, \cdot\right)\left(c f\right.$. [4]); thus, the star $M^{*}$ of a matrix $M$ over this semiring is well-founded. Likewise, $\left(\mathbb{K}\left\langle\left\langle A^{*}\right\rangle\right\rangle,+, \odot\right)$ is also Conway (since every operation is entrywise), and for every matrix $M$ in $\mathbb{K}\left\langle\left\langle A^{*}\right\rangle\right\rangle^{n \times n}, M^{\circledast}$ is the star of $M$ in this semiring.

We prove now that the series realized by a two-way $\mathbb{K}$-representation can be characterized as the unique fixed-point of a system.

Theorem 13. Let $\mathbb{K}$ be a Conway semiring. Let $\rho=(I, \mu, \diamond, T)$ be a two-way $\mathbb{K}$-representation and let $M=\sum_{a \in A} \mu(a)$. Then the unique solution of the system

$$
\left\{\begin{array}{l}
X=\overleftrightarrow{\diamond}+\overleftrightarrow{M} \cdot \mathrm{A}^{*}+(\vec{M} \cdot X) \odot(\overleftrightarrow{M} \cdot X)^{\circledast} \odot\left(\overleftarrow{M} \cdot \mathrm{A}^{*}\right) \\
Y=T+\overleftrightarrow{M} \cdot Y+(\vec{M} \cdot X) \odot(\overleftrightarrow{M} \cdot X)^{\circledast} \odot(\overleftrightarrow{M} \cdot Y)
\end{array}\right.
$$

is $(X, Y)=(\overleftrightarrow{S}, \vec{S} \cdot T)$, with

$$
S=\sum_{w \in A^{*}}(\mu(w) \beth \diamond) w
$$

The series realized by the representation is $I .(\overleftrightarrow{S} \cdot \overleftrightarrow{\diamond})^{\circledast} \odot(\vec{S} . T)$

Proof. Assume first that there exists a solution $\left(X_{0}, Y_{0}\right)$. We first prove by induction that $X_{0}=$ $\overleftrightarrow{S}$. The matrix $M$ is proper, hence $\left\langle X_{0}, \varepsilon\right\rangle=\overleftrightarrow{\diamond}=\langle\overleftrightarrow{S}, \varepsilon\rangle$, and for every letter $a,\left\langle X_{0}, a\right\rangle=$ $\langle\overleftrightarrow{M}, a\rangle=\overleftrightarrow{\mu}(a)=\langle\overleftrightarrow{S}, a\rangle$. Let $w$ be a word such that $\left\langle X_{0}, w\right\rangle=\langle\overleftrightarrow{S}, w\rangle$. Then, for every letter $a$

$$
\begin{aligned}
& \left\langle X_{0}, a w\right\rangle=\left\langle\overleftrightarrow{M} \cdot \mathrm{A}^{*}, a w\right\rangle+\left\langle\vec{M} \cdot X_{0}, a w\right\rangle \cdot\left\langle\overleftrightarrow{M} \cdot X_{0}, a w\right\rangle^{*} \cdot\left\langle\overleftarrow{M} \cdot \mathrm{A}^{*}, a w\right\rangle \\
& =\langle\overleftrightarrow{M}, a\rangle+\langle\vec{M}, a\rangle \cdot\left\langle X_{0}, w\right\rangle \cdot\left(\langle\overleftrightarrow{M}, a\rangle \cdot\left\langle X_{0}, w\right\rangle\right)^{*} \cdot\langle\overleftarrow{M}, a\rangle \\
& =\langle\overleftrightarrow{M}, a\rangle+\langle\vec{M}, a\rangle \cdot\left\langle X_{0}, w\right\rangle \cdot\left(\langle\overleftrightarrow{M}, a\rangle \cdot\left\langle X_{0}, w\right\rangle\right)^{*} \cdot\langle\overleftarrow{M}, a\rangle \\
& =\langle\overleftrightarrow{M}, a\rangle+\langle\vec{M}, a\rangle \cdot\langle\overleftrightarrow{S}, w\rangle \cdot(\langle\overleftrightarrow{M}, a\rangle \cdot\langle\overleftrightarrow{S}, w\rangle)^{*} \cdot\langle\overleftarrow{M}, a\rangle
\end{aligned}
$$

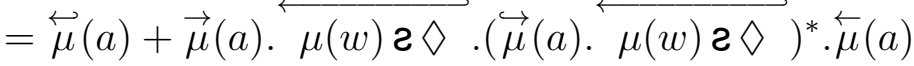

$$
\begin{aligned}
& =\mu \overleftrightarrow{(a) \curvearrowright \mu(w) \circlearrowright} \diamond=\overleftrightarrow{\mu(a w) 乞 \diamond}=\langle\overleftrightarrow{S}, a w\rangle
\end{aligned}
$$


We then prove by induction that $Y_{0}=\vec{S} \cdot T \cdot\left\langle Y_{0}, \varepsilon\right\rangle=T=\overrightarrow{\mu(\varepsilon) \text { ¿ } \diamond} \cdot T=\langle\vec{S} \cdot T, \varepsilon\rangle$. Let $w$ be a word such that $\left\langle Y_{0}, w\right\rangle=\langle\vec{S} . T, w\rangle$. Then, for every letter $a$,

$$
\begin{aligned}
& \left\langle Y_{0}, a w\right\rangle=\left\langle\vec{M} \cdot Y_{0}, a w\right\rangle+\left\langle\vec{M} \cdot X_{0}, a w\right\rangle \cdot\left\langle\overleftrightarrow{M} \cdot X_{0}, a w\right\rangle^{*} \cdot\left\langle\overleftarrow{M} \cdot Y_{0}, a w\right\rangle \\
& =\langle\vec{M}, a\rangle \cdot\left\langle Y_{0}, w\right\rangle+\langle\vec{M}, a\rangle \cdot\left\langle X_{0}, w\right\rangle \cdot\left(\langle\overleftrightarrow{M}, a\rangle \cdot\left\langle X_{0}, w\right\rangle\right)^{*} \cdot\langle\overleftarrow{M}, a\rangle \cdot\left\langle Y_{0}, w\right\rangle \\
& =\langle\vec{M}, a\rangle \cdot\langle\vec{S} \cdot T, w\rangle+\langle\vec{M}, a\rangle \cdot\langle\overleftrightarrow{S}, w\rangle \cdot(\langle\overleftrightarrow{M}, a\rangle \cdot\langle\overleftrightarrow{S}, w\rangle)^{*} \cdot\langle\overleftarrow{M}, a\rangle \cdot\langle\vec{S} \cdot T, w\rangle \\
& =\vec{\mu}(a) . \overrightarrow{\mu(w)} \iota \diamond . T
\end{aligned}
$$

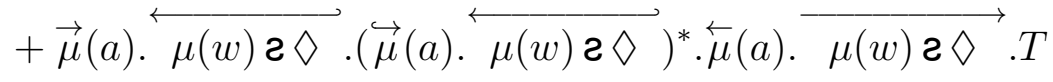

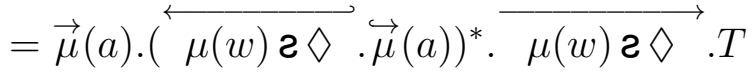

$$
\begin{aligned}
& =\mu \overrightarrow{(a) \circlearrowright \mu(w) \circlearrowright} \diamond=\overrightarrow{\mu(a w) \circlearrowright \diamond} \cdot T=\langle\vec{S} \cdot T, a w\rangle \text {. }
\end{aligned}
$$

It is easy to check that $(\overleftrightarrow{S}, \vec{S} . T)$ is actually a solution to the system.

Finally, for every word $w$,

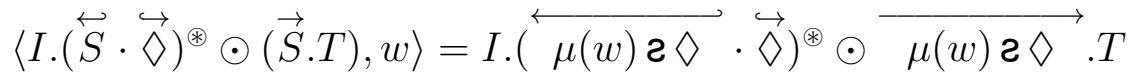

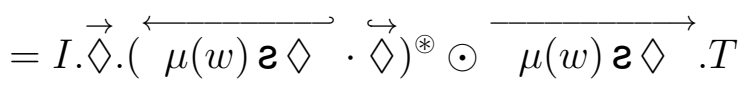

$$
\begin{aligned}
& =I . \overrightarrow{\curvearrowright \mu(w) \curvearrowright \diamond} \cdot T \text {. }
\end{aligned}
$$

EXAmPLE. Let $\rho=(I, \mu, \diamond, T)$ be the two-way $(\mathbb{N} \cup\{\infty\})$ representation defined by:

$$
\begin{gathered}
I=\left[\begin{array}{ll}
1 & 0
\end{array}\right], T=\left[\begin{array}{l}
1 \\
0
\end{array}\right] . \\
\mu(a)=\left[\begin{array}{ll|l}
0 & 1 & 0 \\
1 & 0 & 0 \\
\hline 0 & 0 & 2
\end{array}\right], \mu(b)=\left[\begin{array}{ll|l}
1 & 0 & 0 \\
0 & 0 & 1 \\
\hline 0 & 1 & 0
\end{array}\right], \diamond=\left[\begin{array}{ll|l}
1 & 0 & 0 \\
0 & 1 & 1 \\
\hline 0 & 1 & 0
\end{array}\right] .
\end{gathered}
$$

The system corresponding to this representation is:

$$
\left\{\begin{array}{l}
X_{1}=\left[\begin{array}{l}
0 \\
1
\end{array}\right]+\left[\begin{array}{c}
0 \\
b A^{*}
\end{array}\right]+\left(\left[\begin{array}{ll}
b & a \\
a & 0
\end{array}\right] \cdot X_{1}\right) \odot\left(\left[\begin{array}{ll}
0 & b
\end{array}\right] \cdot X_{1}\right)^{\circledast} \odot\left[2 a A^{*}\right], \\
Y_{1}=\left[\begin{array}{l}
1 \\
0
\end{array}\right]+\left[\begin{array}{ll}
b & a \\
a & 0
\end{array}\right] \cdot Y_{1}+\left(\left[\begin{array}{ll}
b & a \\
a & 0
\end{array}\right] \cdot X_{1}\right) \odot\left(\left[\begin{array}{ll}
0 & b
\end{array}\right] \cdot X_{1}\right)^{\circledast} \odot\left(\left[\begin{array}{ll}
0 & b
\end{array}\right] \cdot Y_{1}\right) .
\end{array}\right.
$$


The last factor of the third term of $X_{1}$ forces words to begin with letter $a$, hence, the product with $\left(\left[\begin{array}{ll}0 & b\end{array}\right] \cdot X_{1}\right)^{\circledast}$ is always null except with the identity; finally, it comes

$$
X_{1}=\left[\begin{array}{c}
0 \\
1+b A^{*}
\end{array}\right]+\left[\begin{array}{cc}
0 & 2 a \\
2 a & 0
\end{array}\right] \cdot X_{1}
$$

Hence, by Arden lemma,

$$
X_{1}=\left[\begin{array}{cc}
0 & 2 a \\
2 a & 0
\end{array}\right]^{*} \cdot\left[\begin{array}{c}
0 \\
1+b(a+b)^{*}
\end{array}\right]=(4 a a)^{*}\left[\begin{array}{c}
2 a \\
1
\end{array}\right]\left(1+b A^{*}\right) .
$$

Likewise, in the third term of $Y_{1}$, the first letter of words must be a $b$, hence,

$$
Y_{1}=\left[\begin{array}{l}
1 \\
0
\end{array}\right]+\left[\begin{array}{ll}
b & a \\
a & 0
\end{array}\right] \cdot Y_{1}+\left[\begin{array}{c}
b 2 a(4 a a)^{*}\left(1+b A^{*}\right) \\
0
\end{array}\right] \odot\left(\left[b(4 a a)^{*}\left(1+b A^{*}\right)\right]\right)^{\circledast} \odot\left(\left[\begin{array}{ll}
0 & b
\end{array}\right] \cdot Y_{1}\right)
$$

Considering the parity of the first block of $a$, it comes

$$
Y_{1}=\left[\begin{array}{l}
1 \\
0
\end{array}\right]+\left[\begin{array}{ll}
b & a \\
a & 0
\end{array}\right] \cdot Y_{1}+\left[\begin{array}{c}
b 2 a(4 a a)^{*}\left(1+b A^{*}\right) \\
0
\end{array}\right] \odot\left(\left[\begin{array}{ll}
0 & b
\end{array}\right] \cdot Y_{1}\right)
$$

We set $Y_{a}=Y_{1} \odot a A^{*}$ and $Y_{b}=Y_{1} \odot\left(1+b A^{*}\right) ; Y_{1}=Y_{a}+Y_{b}$ and it holds

$$
\begin{aligned}
& \left\{\begin{array}{l}
Y_{a}=\left[\begin{array}{ll}
0 & a \\
a & 0
\end{array}\right] \cdot\left(Y_{a}+Y_{b}\right) \\
Y_{b}=\left[\begin{array}{l}
1 \\
0
\end{array}\right]+\left[\begin{array}{ll}
b & 0 \\
0 & 0
\end{array}\right] \cdot\left(Y_{a}+Y_{b}\right)+b\left[\begin{array}{c}
2 a(4 a a)^{*}\left(1+b A^{*}\right) \\
0
\end{array}\right] \odot\left(\left[\begin{array}{ll}
0 & 1
\end{array}\right] \cdot Y_{a}\right)
\end{array}\right.
\end{aligned}
$$

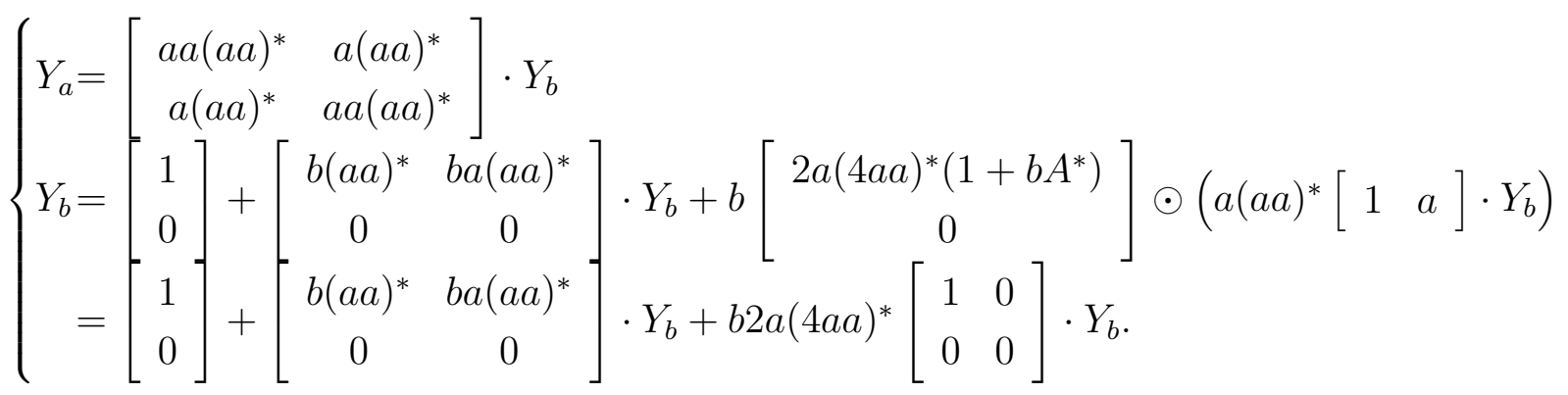

therefore,

$$
Y_{b}=\left[\begin{array}{c}
\left(b(a a)^{*}+b 2 a(4 a a)^{*}\right)^{*} \\
0
\end{array}\right]
$$

The solution of this system is

$$
\left\{\begin{array}{l}
X_{1}=\left[\begin{array}{c}
2 a(4 a a)^{*}\left(1+b A^{*}\right) \\
(4 a a)^{*}\left(1+b A^{*}\right)
\end{array}\right] \\
Y_{1}=\left[\begin{array}{c}
(a a)^{*}\left(b(a a)^{*}+b 2 a(4 a a)^{*}\right)^{*} \\
a(a a)^{*}\left(b(a a)^{*}+b 2 a(4 a a)^{*}\right)^{*}
\end{array}\right] .
\end{array}\right.
$$


Hence, the series realized by the representation is

$$
\begin{aligned}
S_{1} & =\left[\begin{array}{l}
1 \\
0
\end{array}\right] \cdot\left[\begin{array}{cc}
0 & 2 a(4 a a)^{*}\left(1+b A^{*}\right) \\
0 & (4 a a)^{*}\left(1+b A^{*}\right)
\end{array}\right]^{\circledast} \odot\left[\begin{array}{c}
(a a)^{*}\left(b(a a)^{*}+b 2 a(4 a a)^{*}\right)^{*} \\
a(a a)^{*}\left(b(a a)^{*}+b 2 a(4 a a)^{*}\right)^{*}
\end{array}\right] \\
& =(a a)^{*}\left(b(a a)^{*}+b 2 a(4 a a)^{*}\right)^{*}+\left(2 a(4 a a)^{*}\left(1+b A^{*}\right)\right) \odot\left(a(a a)^{*}\left(b(a a)^{*}+b 2 a(4 a a)^{*}\right)^{*}\right) \\
& =\left((a a)^{*}+2 a(4 a a)^{*}\right)\left(b(a a)^{*}+b 2 a(4 a a)^{*}\right)^{*} .
\end{aligned}
$$

Theorem 13 is an implicit characterization of the series realized by a two-way representation. Unfortunately, Equation (1) is not easy to solve (and does not always accept solutions in $\mathbb{K R H} A^{*}$ ). In the next section, we solve it in the particular case of sweeping representations.

\section{Rotating and sweeping representations}

In this section, we characterize series realized by rotating and sweeping representations and we show that they can be denoted by explicit expressions involving rational, entrywise and mirror operators.

Theorem 14. Let $\mathbb{K}$ be a Conway semiring. Then, a series can be realized by a rotating $\mathbb{K}$ representation if and only if it is in $\mathbb{K H a d} A^{*}$. Moreover, let $\rho=(I, \mu, \diamond, T)$ be a rotating $\mathbb{K}$-representation and let $M=\sum_{a \in A} \mu(a) a$, then

$$
|\rho|=I \cdot\left(\vec{M}^{*} \cdot \overleftrightarrow{\diamond} \cdot \overleftrightarrow{\diamond}\right)^{\circledast} \odot \vec{M}^{*} \cdot T .
$$

Proof. Proposition 11 states that every series in $\mathbb{K} H a d A^{*}$ can be realized by a rotating $\mathbb{K}$ representation. Conversely, since $\rho$ is rotating, Equation (1) reduces to

$$
\left\{\begin{array}{l}
X=\overleftrightarrow{\diamond}+\vec{M} \cdot X \\
Y=\mathrm{ld}+\vec{M} \cdot Y
\end{array}\right.
$$

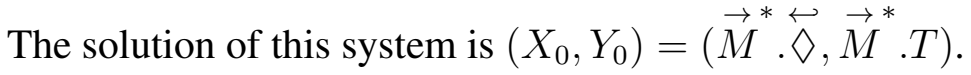

Theorem 15. Let $\mathbb{K}$ be a Conway semiring. Then, a series can be realized by a sweeping $\mathbb{K}$ representation if and only if it is in $\mathbb{K M i r H a d} A^{*}$. Moreover, let $\rho=(I, \mu, \diamond, T)$ be a sweeping $\mathbb{K}$-representation and let $M=\sum_{a \in A} \mu(a) a$, then

$$
|\rho|=I \cdot\left(\left(\vec{M}^{*} \cdot \overleftrightarrow{\diamond}\right) \odot\left(\overleftarrow{M}^{*} \cdot \overleftrightarrow{\nabla}\right)\right)^{\circledast} \odot \vec{M}^{*} \cdot T
$$

Proof. Proposition 11 states that every series in $\mathbb{K} M i r H a d A^{*}$ can be realized by a sweeping $\mathbb{K}$ representation. Conversely, since $\rho$ is sweeping, Equation (1) reduces to

$$
\begin{cases}X= & \overleftrightarrow{\diamond}+(\vec{M} \cdot X) \odot\left(\overleftarrow{M} \cdot \mathrm{A}^{*}\right) \\ Y= & \mathrm{ld}+\vec{M} \cdot Y\end{cases}
$$


It immediatly comes $Y=\vec{M}^{*} . T$, and we prove by induction on the length of words that, for every word $w$,

$$
\langle X, w\rangle=\left\langle\left(\vec{M}^{*} \cdot \overleftrightarrow{\diamond}\right) \odot \overleftarrow{M}^{*}, w\right\rangle
$$

It is true when $w=\varepsilon(\vec{M}$ and $\overleftarrow{M}$ are proper), and if it is true for a word $w$, then, for every letter $a$

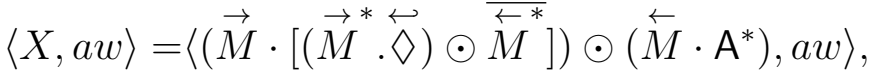

$$
\begin{aligned}
& =\left\langle\vec{M} \cdot\left[\left(\vec{M}^{*} \cdot \overleftrightarrow{\diamond}\right) \odot \overleftarrow{M}^{*}\right], a w\right\rangle \cdot\left\langle\overleftarrow{M} \cdot \mathrm{A}^{*}, a w\right\rangle
\end{aligned}
$$

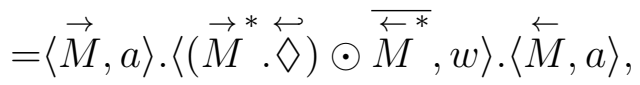

$$
\begin{aligned}
& =\langle\vec{M}, a\rangle \cdot\left\langle\vec{M}^{*} \cdot \overleftrightarrow{\diamond}, w\right\rangle \cdot\left\langle\overleftarrow{M}^{*}, \bar{w}\right\rangle \cdot\langle\overleftarrow{M}, a\rangle \\
& =\left\langle\left(\mathrm{ld}+\vec{M} \cdot \vec{M}^{*}\right) \cdot \overleftrightarrow{\diamond}, a w\right\rangle \cdot\left\langle\left(\mathrm{ld}+\overleftarrow{M}^{*} \cdot \overleftarrow{M}\right), \overline{a w}\right\rangle \\
& =\left\langle\vec{M}^{*} \cdot \overleftrightarrow{\diamond}, a w\right\rangle \cdot\left\langle\overleftarrow{M}^{*}, a w\right\rangle, \\
& =\left\langle\left(\vec{M}^{*} \cdot \overleftrightarrow{\nabla}\right) \odot \overleftarrow{M}^{*}, a w\right\rangle
\end{aligned}
$$

Finally, $X=\left(\vec{M}^{*} . \overleftrightarrow{\diamond}\right) \odot \overleftarrow{M}^{*}$. Hence, by Theorem 13, $\mathcal{A}$ realizes the series

$$
S=I \cdot\left(\left(\vec{M}^{*} \cdot \overleftrightarrow{\diamond}\right) \odot \overleftarrow{M}^{*} \cdot \overleftrightarrow{\diamond}\right)^{\circledast} \odot \vec{M}^{*} \cdot T
$$

Since the star of matrices can be effectively computed by the usual formulae, these theorems state that, for rotating and sweeping representations, an explicit expression (involving rational, entrywise and mirror operators) representing the realized series can be computed.

EXAMPLE. Let $\rho$ be the following sweeping representation:

$$
\begin{array}{cc}
I=\left[\begin{array}{cc}
1 & 0
\end{array}\right], \quad T=\left[\begin{array}{c}
0 \\
1 / 2
\end{array}\right], & \overleftrightarrow{\diamond}=\left[\begin{array}{cc}
0 & 0 \\
1 / 2 & 0
\end{array}\right], \quad \overleftrightarrow{\diamond}=\left[\begin{array}{ll}
0 & 0 \\
1 & 0
\end{array}\right] \\
\vec{M}=\left[\begin{array}{cc}
a+\frac{1}{2} b & \frac{1}{2} b \\
0 & a+b
\end{array}\right] & \overleftarrow{M}=\left[\begin{array}{cc}
\frac{1}{2} a+b & \frac{1}{2} a \\
0 & a+b
\end{array}\right] .
\end{array}
$$

It holds:

$\vec{M}^{*}=\left[\begin{array}{cc}\left(a+\frac{1}{2} b\right)^{*} & \left(a+\frac{1}{2} b\right)^{*} \frac{1}{2} b(a+b)^{*} \\ 0 & (a+b)^{*}\end{array}\right], \quad \overleftarrow{M}^{*}=\left[\begin{array}{cc}\left(\frac{1}{2} a+b\right)^{*} & (a+b)^{*} \frac{1}{2} a\left(\frac{1}{2} a+b\right)^{*} \\ 0 & (a+b)^{*}\end{array}\right]$ 
Therefore, the series realized by $\rho$ is:

$$
\begin{aligned}
S_{2} & =\left[\begin{array}{ll}
1 & 0
\end{array}\right] \cdot\left(\left[\begin{array}{cc}
\frac{1}{2}\left(a+\frac{1}{2} b\right)^{*} \frac{1}{2} b(a+b)^{*} & 0 \\
\frac{1}{2}(a+b)^{*} & 0
\end{array}\right] \odot\left[\begin{array}{cc}
(a+b)^{*} \frac{1}{2} a\left(\frac{1}{2} a+b\right)^{*} & 0 \\
(a+b)^{*} & 0
\end{array}\right]\right) \\
\odot\left[\begin{array}{c}
\left(a+\frac{1}{2} b\right)^{*} \frac{1}{2} b(a+b)^{*} \\
(a+b)^{*}
\end{array}\right] & \\
& =\left(\left(\frac{1}{4}\left(a+\frac{1}{2} b\right)^{*} b(a+b)^{*}\right) \odot\left(\frac{1}{2}(a+b)^{*} a\left(\frac{1}{2} a+b\right)^{*}\right)\right)^{\circledast} \odot\left(\left(a+\frac{1}{2} b\right)^{*} \frac{1}{2} b(a+b)^{*}\right) \\
& =\left(\frac{1}{8}\left(a+\frac{1}{2} b\right)^{*}\left(b(a+b)^{*} a+a\left(\frac{1}{2} a+\frac{1}{2} b\right)^{*} b\right)\left(\frac{1}{2} a+b\right)^{*}\right)^{\circledast} \odot\left(\left(a+\frac{1}{2} b\right)^{*} \frac{1}{2} b(a+b)^{*}\right) .
\end{aligned}
$$

\section{Two-Way Automata}

\subsection{Definition of Two-Way Automata}

We define weighted two-way automata that are extensions of classical two-way automata. We consider, like for instance in [3], that, during the computation of two-way automata, the move on the input only depends on the state and not on the transition. These two-way automata are as powerful as the usual ones; they correspond to the family of $\delta$-local automata defined in [5].

Definition 3. Let $\mathbb{K}$ be a semiring, $A$ an alphabet and $\square$ a special symbol, called endmarker, which does not belong to $A$. A two-way $\mathbb{K}$-automaton over $A$ is a tuple $\left(Q_{+}, Q_{-}, A, \mathbb{K}, E, I, T\right)$, where

$-Q=Q_{+} \cup Q_{-}$is a finite set of states;

$-\delta: Q \longrightarrow\{-1,+1\}$ is the direction of every state;

- E : $Q \times A \times Q \cup Q_{+} \times \square \times Q_{-} \cup Q_{-} \times \square \times Q_{+} \longrightarrow \mathbb{K}$ is the transition function;

$-I: Q_{+} \longrightarrow \mathbb{K}$ is the initial function;

$-T: Q_{+} \longrightarrow \mathbb{K}$ is the final function.

The set of transitions is the support of $E$, the set of initial states is the support of $I$ and the set of final states is the support of $T$. For every transition $e=(p, a, q), \lambda(e)=a$ is the label of $e$, $\sigma(e)=p$ is the source, and $\tau(e)=q$ is the target of $e$.

Let $\delta: Q \longrightarrow\{-1,+1\}$ defined by $\delta(p)=+1$ for every $p$ in $Q_{+}$and $\delta(p)=-1$ for every $p$ in $Q_{-}$. A path in such an automaton is a sequence $\left(e_{i}, p_{i}\right)_{i \in[0 ; k]}$, where every $e_{i}$ is a transition and every $p_{i}$ is a non negative integer such that:

- for every $i$ in $[1 ; k], \sigma\left(e_{i}\right)=\tau\left(e_{i-1}\right)$,

- for every $i$ in $[1 ; k], p_{i}=p_{i-1}+\delta\left(\sigma\left(e_{i}\right)\right)$.

A word $w=w_{0} \ldots w_{n}$ is admissible for such a path if, for every $i$ in $[0 ; k], p_{i}$ is in $[0 ; n]$ and $\lambda\left(e_{i}\right)=w_{p_{i}}$.

It is strongly admissible for the path if it is admissible and:

$-p_{0}=0$ if $\sigma\left(e_{0}\right)$ is in $Q_{+}$and $p_{0}=n$ if $\sigma\left(e_{0}\right)$ is in $Q_{-}$;

$-p_{k}=n$ if $\tau\left(e_{k}\right)$ is in $Q_{+}$and $p_{k}=0$ if $\tau\left(e_{k}\right)$ is in $Q_{-}$. 


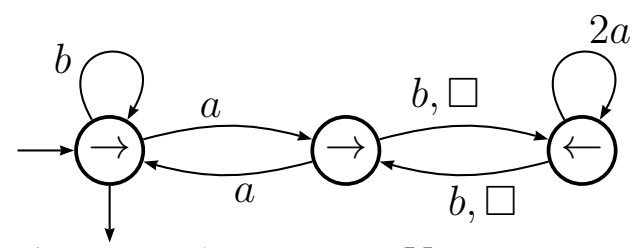

Figure 1: The two-way $\mathbb{N}$-automaton $\mathcal{A}_{1}$

A path $\pi=\left(e_{i}, p_{i}\right)_{i \in[0 ; k]}$ is a computation on the word $w=w_{1} \ldots w_{n}$ of $A^{*}$, if $-\square w \square$ is admissible for $\pi$,

$-p_{0}=1$ and $\sigma\left(e_{0}\right)$ is an initial state,

$-p_{k}=n$ and $\tau\left(e_{k}\right)$ is a final state.

The weight of a computation $\pi=\left(e_{i}, p_{i}\right)_{i \in[0 ; k]}$ is the product

$$
\mathrm{w}(\pi)=I\left(\sigma\left(e_{0}\right)\right) \cdot\left(\prod_{i=0}^{k} E\left(e_{i}\right)\right) \cdot T\left(\tau\left(e_{k}\right)\right) .
$$

Notice that the weight of a computation is computed "one-way", like the trace of a two-way computation defined in [11].

EXAMPLE. The two-way $\mathbb{N}$-automaton of Figure 1 accepts every word. It is a deterministic automaton: there is only one initial state, for every letter in input and every state, there is at most one transition, and there is no transition with label $\square$ that leaves a final state. Every word with $k$ letters $a$ in blocks of odd lengths is accepted with a weight equal to $2^{k}$.

Behaviour of a two-way $\mathbb{K}$-automaton. A two-way $\mathbb{K}$-automaton $\mathcal{A}$ is valid, if, for every word $w$, the sum of the weights of the computations on $w$ is defined. In this case, we denote $\langle|\mathcal{A}|, w\rangle$ this sum and, the behaviour of $\mathcal{A}$ is the formal power series

$$
|\mathcal{A}|=\sum_{w \in A^{*}}\langle|\mathcal{A}|, w\rangle w
$$

We also say that the series $|\mathcal{A}|$ is realized by $\mathcal{A}$.

Classes of two-way $\mathbb{K}$-automata. We define here subclasses of two-way automata that correspond to subclasses of two-way representations described above.

Let $\mathcal{A}=\left(Q_{+}, Q_{-}, A, \mathbb{K}, E, I, T\right)$ be a two-way $\mathbb{K}$-automaton.

- $\mathcal{A}$ is one-way if $Q_{-}=\emptyset$.

- $\mathcal{A}$ is sweeping if every half-turn is labeled by the endmarker: for every transition $e$, $\delta(\sigma(e)) \neq \delta(\tau(e))$ implies $\lambda(e)=\square$

- $\mathcal{A}$ is rotating if it is sweeping and, for every $p$ in $Q_{-}$, for every letter $a$, there is a unique transition with source $p$ and label $a$; this transition is a loop on $p$ with weight 1 .

Thus, depending on the state reached after reading the input, the automaton can rewind to the beginning of the input. 


\subsection{Representation of a two-way automaton.}

Let $\mathbb{K}$ be a Conway semiring and let $\mathcal{A}=\left(Q_{+}, Q_{-}, A, \mathbb{K}, E, I, T\right)$ be a two-way $\mathbb{K}$-automaton. The representation of $\mathcal{A}$ is the two-way representation $(I, \mu, \diamond, T)$ of size $Q_{+}+Q_{-}$, where

$$
\begin{aligned}
\forall a \in A, \forall(p, q) \in Q \times Q, \mu(a)_{p, q}=E(p, a, q) \\
\forall(p, q) \in Q \times Q, \nabla_{p, q}= \begin{cases}E(p, \square, q) & \text { if } \delta(p) \neq \delta(q), \\
1 & \text { if } p \in Q_{+} \text {and } p=q, \\
0 & \text { otherwise. }\end{cases}
\end{aligned}
$$

In the case where $\mathbb{K}$ is the Boolean semiring, the monoid $\mathcal{M}$ generated by $\left\{\mu(a) \mid a \in A^{*}\right\}$ (with 2 as product) is the finite monoid of the two-way automaton, defined in [2]; $\mu$ is a morphism from $A^{*}$ into $\mathcal{M}$, and the language accepted by the automaton is $\mu^{-1}(P)$, where $P=\{M \in \mathcal{M} \mid$ I. $\diamond 2 M \curvearrowright \diamond . T=1\}$.

In general, the Conway properties does not tell how the sum of the weights of all computations labeled by a given word can be computed in the two-way automaton. To state the equivalence between the automaton and its representation, we must restrain to a framework where the star and the infinite sums are related.

EXAMPLE. We consider the series

$$
\sum_{k \geqslant 0} \frac{a^{k}}{k+1}=\left(a^{*}-\left(\frac{a}{2}\right)^{*} \cdot\left(\frac{a}{2}\right)^{*}\right)^{\circledast} \odot\left(\frac{a}{2}\right)^{*} .
$$

We can apply the construction of Proposition 9 to build a two-way $\mathbb{Q}$-automaton from this expression. The result is the automaton $\mathcal{A}_{2}$ of Figure 2 (left). This automaton is not valid: for every word $w$, there are an infinite number of computations with label $w$ and weight $\frac{1}{2^{|w|}}$. Nevertheless, there exists a two-way $\mathbb{Q}$-automaton corresponding to the representation that realizes this series; it is drawn on Figure 2 (right), and this one is valid. Since the weight of every computation is positive, one can perform block sums and therefore the sum of the weights of the computations labeled by a given word exists and is the value given by the representation.

\subsection{Simple Two-Way Automata}

We say that a two-way automaton is simple if for every input, the number of computations is finite. Obviously, a simple two-way weighted automaton is always valid and we need no assumption on the semiring of weights to handle simple automata.

Definition 4. Let $\mathcal{A}=\left(Q_{+}, Q_{-}, A, \mathbb{K}, E, I, T\right)$ be a two-way automaton. A path $\pi=\left(e_{i}, p_{i}\right)_{i \in[0 ; k]}$ is an unmoving circuit in $\mathcal{A}$ if $\sigma\left(e_{0}\right)=\tau\left(e_{k}\right), p_{0}=p_{k}+\delta\left(\sigma\left(e_{0}\right)\right)$, and there exists an admissible word for $\pi$.

Lemma 16. [1] A two-way automaton is simple if and only if there is no computation containing an unmoving circuit. 

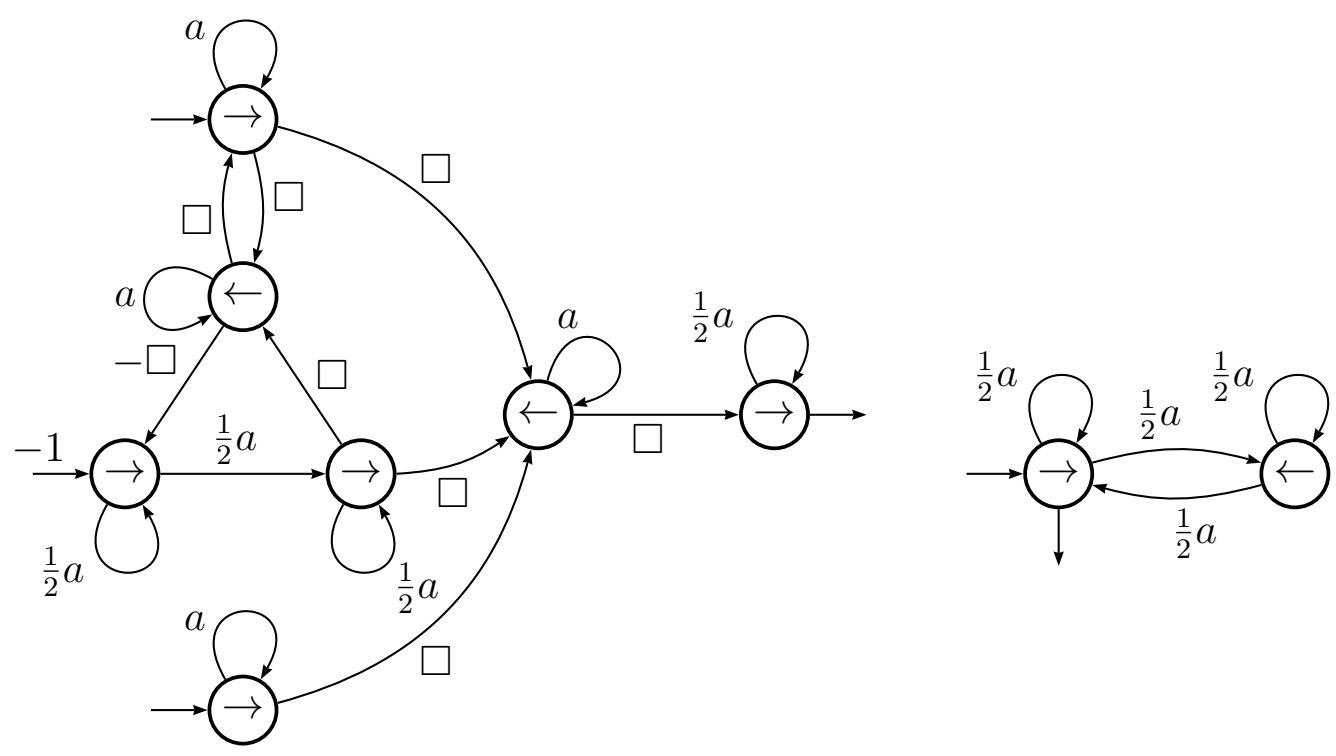

Figure 2: The sweeping $\mathbb{Q}$-automaton $\mathcal{A}_{2}$ and the two-way $\mathbb{Q}$-automaton $\mathcal{A}_{3}$.

Proposition 17. [1] It is decidable whether a two-way automaton is simple.

These results are obtained through the study of the crossing sequences, that are the sequences of states of a computations meet at the same position. A one-way automaton can emulate the sequence of crossing sequences on an input. This is the idea of the original proof of the equivalence between 1NFA and 2NFA [16]. If $\mathbb{K}$ is commutative, the computation of the value can also be emulated; finally:

Proposition 18. [1] If $\mathbb{K}$ is a commutative semiring, every simple two-way automaton realizes a rational series.

Like for unweighted automata, simple two-way weighted automata can nevertheless be more succint that one-way weighted automata.

Particular simple two-way automata are deterministic automata.

Definition 5. A two-way automaton is deterministic if

- it contains at most one initial state,

- for every letter (or endmarker) a and every state $p$, there is at most one transition e with $\sigma(e)=p$ and $\lambda(e)=a$,

- for every final state $p$, there is no transition e with $\sigma(e)=p$ and $\lambda(e)=\square$.

In [5], it is proven that a deterministic two-way weighted automaton can emulate any unambiguous one-way weighted automaton i.e. a one-way weighted automaton with only one computation for each accepted word.

Simple two-way weighted automata are always valid, but the use of their representation is not always accurate. 
EXAMPLE. The representation of the automaton $\mathcal{A}_{1}$ of Figure 1 is:

$$
\begin{gathered}
I=\left[\begin{array}{ll}
1 & 0
\end{array}\right], \quad T=\left[\begin{array}{l}
1 \\
0
\end{array}\right] \\
\diamond=\left[\begin{array}{ll|l}
1 & 0 & 0 \\
0 & 1 & 1 \\
\hline 0 & 1 & 0
\end{array}\right], \quad \mu(a)=\left[\begin{array}{ll|l}
0 & 1 & 0 \\
1 & 0 & 0 \\
\hline 0 & 0 & 2
\end{array}\right], \quad \mu(b)=\left[\begin{array}{ll|l}
1 & 0 & 0 \\
0 & 0 & 1 \\
\hline 0 & 1 & 0
\end{array}\right] .
\end{gathered}
$$

For instance, to compute the weight of $a b$, on can compute

$$
\begin{aligned}
& \mu(a b)=\left[\begin{array}{cc|c}
0 & 0 & 2 \\
1 & 0 & 0 \\
\hline 0 & 1 & 0
\end{array}\right], \quad \diamond \boldsymbol{\imath} \mu(a b)=\left[\begin{array}{cc|c}
2 & 0 & 0 \\
1 & 0 & 1 \\
\hline 0 & 1 & 0
\end{array}\right]
\end{aligned}
$$

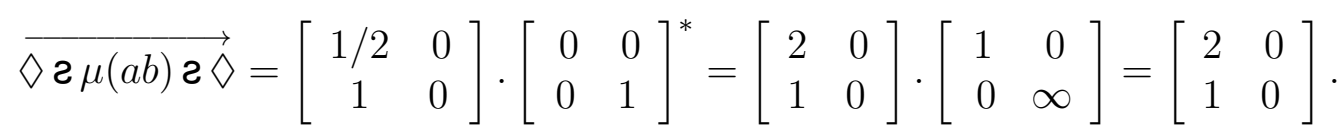

Hence, the weight of $a b$ is 2 . This value can be computed direcly with the automaton, since it is deterministic. Notice that in the computations with the representation, non trivial star operations may appear. Actually, starting from the state in the center of the figure, one can follow a unmoving circuit with admissible word $b b$ (or $b \square$ ).

This automaton is valid in $\mathbb{N}$, but to perform the evalutation through its representation, we must embed $\mathbb{N}$ into the Conway semiring $\mathbb{N} \cup\{\infty\}$.

The same automaton with weight in $\mathbb{Z}$ (replace 2 by -2 ) is always valid, but there is no embedding of $\mathbb{Z}$ into a Conway semiring and there is no more correct representation for this automaton.

\subsection{Two-Way Automata over Rationally Additive Semirings}

We consider now rationally additive semirings introduced in [8], and we prove in this framework the equivalence between two-way weighted automata and two-way representations.

Definition 6. [8] A semiring $\mathbb{K}$ is a rationally additive semiring if it is equipped with an operator $\sum$ defined on some countable families such that, for every countable set I and every family $s$ in $\mathbb{K}^{I}$

1. if I is finite, $\sum_{i \in I} s_{i}$ exists and is the sum of elements of $s$;

2. for every element $x$ of $\mathbb{K}, \sum_{i \in \mathbb{N}} x^{i}$ exists and $x^{*}$ is defined as $x^{*}=\sum_{i \in \mathbb{N}} x^{i}$.

3. for every element $x$ of $\mathbb{K}$, if $\sum s$ exists, so do $\sum_{i \in I} x . s_{i}$ and $\sum_{i \in I} s_{i} . x$, and

$$
\sum_{i \in I} x . s_{i}=x \cdot\left(\sum s\right) \quad \text { and } \sum_{i \in I} s_{i} \cdot x=\left(\sum s\right) \cdot x
$$


4. assume that $I$ is the disjoint union of sets $\left(I_{j}\right)_{j \in J}$; if for every $j$ in $J, r_{j}=\sum_{i \in I_{j}} s_{i}$ exists, and $\sum_{j \in J} r_{j}$ exists, then $\sum s$ exists and $\sum s=\sum_{j \in J} r_{j}$;

5. assume that $I$ is the disjoint union of sets $\left(I_{j}\right)_{j \in J}$; if for every $j$ in $J, r_{j}=\sum_{i \in I_{j}} s_{i}$ exists, and $\sum s$ exists, then for every $j$ in $J, \sum_{j \in J} r_{j}$ exists and $\sum_{j \in J} r_{j}=\sum s$.

EXAMPLE. Many positive semirings are rationally additive or can be completed to be rationally additive, for instance $\mathbb{N} \cup\{\infty\}, \mathbb{Q}_{+} \cup\{\infty\},(\mathbb{N} \cup\{\infty\}$, min, +$)$, the regular languages, every complete lattice, or every positive finite semiring. Nevertheless, many current semirings are not rationally additive. For instance, no ring is a rationally additive semiring; otherwise $s=$ $\sum_{n=0}^{\infty}(-1)^{n}$ exists, then $s=0$ by block sum, and $s=1+(-1) . s$, which is a contradiction. The study of two-way automata and representions in semirings that are not rationally additive requires further investigation. Specific cases have already been studied: $\mathbb{Z}$ in [1], or $(\mathbb{Z} \cup\{\infty\}$, min, +$)$ in $[6]$.

Proposition 19. [8, Prop. 4] A rationally additive semiring is a Conway semiring.

This means that, in rationally additive semirings, the combinatorial approach of star (as sum of powers) and the axiomatic approach meet. As a consequence, we show that, in this framework, the combinatorial description of series by two-way automata is equivalent to the algebraic description by two-way representations.

Proposition 20. Let $\mathbb{K}$ be a rationally additive semiring, and let $\rho=(I, \mu, \diamond, T)$ be the representation of a two-way $\mathbb{K}$-automaton $\mathcal{A}$. For every non empty word $w=w_{0} \ldots w_{n-1}$ of $A^{*}$, and every pair of states $p, q$, let $P(w)_{p, q}$ be the set of paths from $p$ to $q$ for which $w$ is strongly admissible. Then, the sum of the weights of $P(w)_{p, q}$ exists and

$$
\sum_{\pi \in P(w)_{p, q}} \mathrm{w}(\pi)=\mu(w)_{p, q} .
$$

Proof. The proof is by induction on the length of $w$. If $n=1, P(w)_{p, q}$ is either empty and there is no transition from $p$ to $q$ with label $a$ in $\mathcal{A}$; in this case, $\mu(w)_{p, q}=0$; or $P(w)_{p, q}$ is the singleton that contains the transition from $p$ to $q$ with label $a$ in $\mathcal{A}$; in this case, the weight of this transition is by definition $\mu(w)_{p, q}$.

Assume that $n>1$, then $w=u a$ where $u$ is a word with length $n-1$ and $a$ is a letter. For every path $\pi=\left(e_{i}, p_{i}\right)_{i \in[0 ; k]}$ in $P(w)_{p, q}$, we set $R_{\pi}=\left\{i \in[0 ; k] \mid p_{i}=n\right\}$. We set $P(w)_{p, q}^{(t)}=\left\{\pi \in P(w)_{p, q}|| R_{\pi} \mid=t\right\}$; obviously $P(w)_{p, q}=\bigcup_{t \in \mathbb{N}} P(w)_{p, q}^{(t)}$. We consider now the (partial) operation of concatenation of paths: let $\pi=\left(e_{i}, p_{i}\right)_{i \in[0 ; k]}$ and $\zeta=\left(f_{i}, q_{i}\right)_{i \in[0 ; \ell]}$ be two paths such that $\tau\left(e_{k}\right)=\sigma\left(f_{0}\right)$, then $\pi . \zeta=\left(g_{i}, r_{i}\right)_{i \in[0 ; k+\ell+1]}$, with

$$
\left(g_{i}, r_{i}\right)= \begin{cases}\left(e_{i}, p_{i}\right) & \text { if } i \leqslant k, \\ \left(f_{i-k-1}, q_{i-k-1}-q_{0}+p_{k}+\delta\left(\sigma\left(f_{0}\right)\right)\right) & \text { if } i>k .\end{cases}
$$


This operation extends to sets of paths.

Assume first that $p$ and $q$ are in $Q_{+}, P(w)_{p, q}^{(0)}$ is empty and $P(w)_{p, q}^{(1)}=\cup_{r \in Q_{+}} P(u)_{p, r} . P(a)_{r, q}$; more generally, for every $t>0$,

$$
P(w)_{p, q}^{(t)}=\bigcup_{\substack{r_{1}, \ldots r_{t} \in Q_{+}, s_{1}, \ldots s_{t-1} \in Q_{-}}} P(u)_{p, r_{1}} \cdot P(a)_{r_{1}, s_{1}} P(u)_{s_{1}, r_{2}} \ldots P(u)_{s_{t-1}, r_{t}} P(a)_{r_{t}, q}
$$

By induction hypothesis, for every pair of states $r, s$, the sum of the weights of paths in $P(u)_{r, s}$ (resp. $\left.P(a)_{r, s}\right)$ is defined, hence, by [8, Prop. 3], the sum of the weights of paths in $P(w)_{p, q}^{(t)}$ is defined and

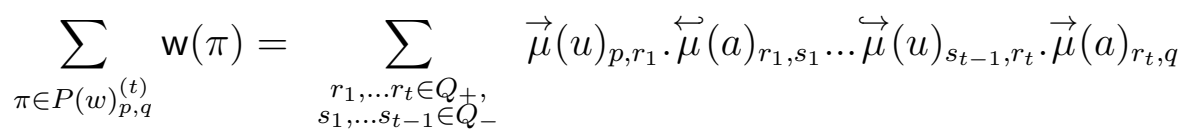

$$
\begin{aligned}
& =\left(\vec{\mu}(u) \cdot(\overleftrightarrow{\mu}(a) \cdot \overleftrightarrow{\mu}(u))^{t-1} \cdot \vec{\mu}(a)\right)_{p, q} .
\end{aligned}
$$

By [8, Th. 9], if $\mathbb{K}$ is a rationally additive semiring, so is the semiring of matrices $\mathbb{K}^{m \times m}$ for every $m$. Therefore, the sum over $t$ of $(\overleftrightarrow{\mu}(a) . \overleftrightarrow{\mu}(u))^{t}$ is defined. Hence, by the fourth axiom of rationally additive semirings, the sum of the weights of paths in $P(w)$ exists and

$$
\sum_{\pi \in P(w)_{p, q}} \mathrm{w}(\pi)=\left(\vec{\mu}(u) \cdot(\overleftrightarrow{\mu}(a) \cdot \overleftrightarrow{\mu}(u))^{*} \vec{\mu}(a)\right)_{p, q}=\vec{\mu}(u a)_{p, q}
$$

Assume now that $p$ is in $Q_{+}$and $q$ is in $Q_{-}$, then $P(w)_{p, q}^{(0)}=P(u)_{p, q}$ and for every positive $t$,

$$
P(w)_{p, q}^{(t)}=\bigcup_{\substack{r_{1}, \ldots r_{t} \in Q_{+}, s_{1}, \ldots s_{t} \in Q_{-}}} P(u)_{p, r_{1}} \cdot P(a)_{r_{1}, s_{1}} P(u)_{s_{1}, r_{2}} \ldots P(a)_{r_{t}, s_{t}} P(u)_{s_{t}, q} .
$$

By the same arguments, the sum of the weights of paths in $P(w)_{p, q}^{(t)}$ exists and

$$
\sum_{\pi \in P(w)_{p, q}^{(t)}} \mathrm{w}(\pi)=\left(\vec{\mu}(u) \cdot(\overleftrightarrow{\mu}(a) \cdot \overleftrightarrow{\mu}(u))^{t-1} \cdot \overleftrightarrow{\mu}(a) \cdot \overleftarrow{\mu}(u)\right)_{p, q}
$$

Finally, we get

$$
\sum_{\pi \in P(w)_{p, q}} \mathrm{w}(\pi)=\overleftrightarrow{\mu}(u)_{p, q}+\left(\vec{\mu}(u) \cdot(\overleftrightarrow{\mu}(a) \cdot \overleftrightarrow{\mu}(u))^{*} \cdot \overleftrightarrow{\mu}(a) \cdot \overleftarrow{\mu}(u)\right)_{p, q}=\overleftrightarrow{\mu}(u a)_{p, q}
$$

The proof is similar in the cases where $p$ is in $Q_{-}$.

Theorem 21. Let $\mathbb{K}$ be a rationally additive semiring and let $\mathcal{A}$ be a two-way $\mathbb{K}$-automaton. Then, the automaton $\mathcal{A}$ is valid and the series realized by $\mathcal{A}$ is the same as the series realized by the representation of $\mathcal{A}$. 
Proof. The proof is similar to the proof of Proposition 20. Let $w=w_{1} \ldots w_{n}$ be a word in $A^{*}$; for every pair of states $p, q$ in $Q_{+}$, let $S(w)_{p, q}$ be the set of paths $\pi=\left(e_{i}, p_{i}\right)_{i \in[0 ; k]}$ from $p$ to $q$ such that $\square w$ is admissible for $\pi, p_{0}=1$ and $p_{k}=n$; like in the proof of Proposition 20, we show that the sum of the weights of $S(w)_{p, q}$ exists and is equal to $(\diamond \boldsymbol{\curvearrowright} \mu(w))_{p, q}$.

Likewise, if $p$ is in $Q_{-}$and $q$ in $Q_{+}$, we set $S(w)_{p, q}$ as the set of paths $\pi=\left(e_{i}, p_{i}\right)_{i \in[0 ; k]}$ from $p$ to $q$ such that $\square w$ is admissible for $\pi, p_{0}=k$ and $p_{k}=n$; the sum of the weights of these paths is equal to $(\diamond \boldsymbol{\imath} \mu(w))_{p, q}$.

Finally, for every pair of states $p, q$ in $Q_{+}, C(w)_{p, q}$ be the set of paths $\pi=\left(e_{i}, p_{i}\right)_{i \in[0 ; k]}$ from $p$ to $q$ such that $\square w \square$ is admissible for $\pi, p_{0}=1$ and $p_{k}=n$; let $R_{\pi}=\left\{i \in[0 ; k] \mid p_{i}=n+1\right\}$ and, for every $t$, let $C(w)_{p, q}^{(t)}=\left\{\pi \in C(w)_{p, q}|| R_{\pi} \mid=t\right\}$. Then, for every $t$,

$$
C(w)_{p, q}^{(t)}=\bigcup_{\substack{r_{1}, \ldots r_{t} \in Q_{+}, s_{1}, \ldots s_{t} \in Q_{-}}} S(w)_{p, r_{1}} \cdot f_{r_{1}, s_{1}} S(w)_{s_{1}, r_{2}} \ldots f_{r_{t}, s_{t}} S(w)_{s_{t}, q}
$$

where every $f_{r_{i}, s_{i}}$ is a transition with label $\square$. Hence, by the same arguments as in the proof of Proposition 20, the sum of the weights of the paths in $C(w)_{p, q}$ exists and

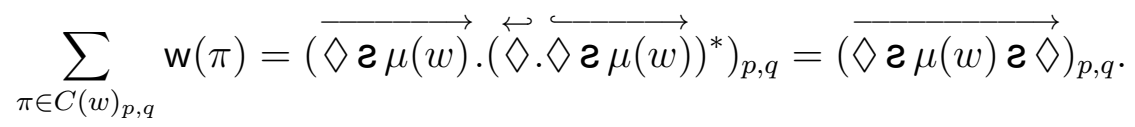

The sum of the weights of the computations with label $w$ that start in an initial state $p$ and end in a final state $q$ is therefore $I_{p} \cdot \vec{\diamond} \mu \mu(w) \mathrm{\iota} \diamond \cdot T_{q}$.

Finally, the sum of the weights of the computations of $\mathcal{A}$ with label $w$ exists and is equal to

$$
I . \diamond 2 \mu(w) \curvearrowright \diamond . T .
$$

EXAMPLE. Let $\mathbb{K}=\mathcal{P}\left(\{x, y\}^{*}\right)$ and let $r$ be the following series over $a^{*}$ :

$$
r=\sum_{k=0}^{\infty}\left(x^{k} y^{k}\right) a^{k} .
$$

This series can be realized by a rotating two-way automaton with the following representation:

$$
\left(\left[\begin{array}{ll}
1 & 0
\end{array}\right], \mu(a)=\left[\begin{array}{ll|l}
x & 0 & 0 \\
0 & y & 0 \\
\hline 0 & 0 & 1
\end{array}\right],\left[\begin{array}{ll|l}
0 & 0 & 1 \\
0 & 0 & 0 \\
\hline 0 & 1 & 0
\end{array}\right],\left[\begin{array}{l}
0 \\
1
\end{array}\right]\right) .
$$

We consider now

$$
r \cdot r=\sum_{k=0}^{\infty} \sum_{i=0}^{k}\left(x^{i} y^{i} x^{k-i} y^{k-i}\right) a^{k} .
$$

Assume that $r \cdot r$ is realized by a two-way automaton $\mathcal{A}$. We consider a computation $\pi$ with label $a^{k}$ such that $v=x^{i} y^{i} x^{k-i} y^{k-i}$ belongs to the weight of $\pi$. After producing $x^{i}$, since the 
automaton is memoryless, it can not store the value of $i$ (if $k$ is larger than the number of states, there are two many possible values for $i$ ); to produce the same number of $y$, the only information that can be used by the automaton is the position in the input, but to take advantage of this information, the automaton must go back to the beginning of the input; it can produces $y^{i}$ but it has lost all the information on $i$ and can therefore not produce $x^{k-i} y^{k-i}$. Likewise $r^{*}$ can not be realized by a two-way automaton $\mathcal{A}$.

This example proves the following proposition:

Proposition 22. The set of two-way recognizable series is not closed under Cauchy product or Kleene star.

Proposition 23. Let $A$ and $B$ be two alphabets, let $\varphi$ be a length-preserving morphism from $A^{*}$ into $B^{*}$, and let $\rho$ be a two-way representation over $A^{*}$. Then

$$
\varphi(|\rho|)=\sum_{w \in A^{*}}\langle|\rho|, w\rangle \varphi(w)
$$

is not necessarily two-way recognizable.

Proof. We use the same example; let $r^{\prime}$ be the following series over $B^{*}=\{a, b\}^{*}$ with coefficients in $\mathcal{P}\left(\{x, y\}^{*}\right)$ :

$$
r^{\prime}=1+\sum_{k=0}^{\infty} \sum_{h=0}^{\infty}\left(x^{k+1} y^{k+1} x^{h} y^{h}\right) a^{k} b a^{h} .
$$

This series is two-way recognizable, but the morphism that maps both $a$ and $b$ onto $a$ sends $r^{\prime}$ to $r \cdot r$ which is not recognizable.

\section{References}

[1] Marcella Anselmo. Two-way automata with multiplicity. In ICALP'90, volume 443 of Lect. Notes in Comput. Sci., pages 88-102, 1990.

[2] Jean-Camille Birget. Concatenation of inputs in a two-way automaton. Theor. Comput. Sci., 63(2):141-156, 1989.

[3] Jean-Camille Birget. Two-way automaton computations. RAIRO Inform. Théor. App., 24:47-66, 1990.

[4] Stephen L. Bloom and Zoltán Ésik. Iteration Theories: The equational logic of iterative processes. Monogr. Theoret. Comput. Sci. EATCS Ser. Springer-Verlag, 1993.

[5] Vincent Carnino and Sylvain Lombardy. On Determinism and Unambiguity of Weighted Two-way Automata. In AFL'14, volume 151 of EPTCS, pages 188-200, 2014.

[6] Vincent Carnino and Sylvain Lombardy. Tropical Two-Way Automata. In TCS'14, volume 8705 of Lect. Notes in Comput. Sci., pages 195-206, 2014. 
[7] John H. Conway. Regular Algebra and Finite Machines. Chapman and Hall, London, 1971.

[8] Zoltán Ésik and Werner Kuich. Rationally additive semirings. J. UCS, 8(2):173-183, 2002.

[9] Michel Fliess. Matrices de hankel. J. Math Pures et Appl., 53:197-222, 1974.

[10] Robert McNaughton and Hisao Yamada. Regular expressions and state graphs for automata. IRE Trans. Electronic Computers, 9:39-47, 1960.

[11] Jean-Pierre Pécuchet. Automates boustrophedon, semi-groupe de birget et monoide inversif libre. RAIRO Inform. Théor. App., 19(1):71-100, 1985.

[12] Giovanni Pighizzini. Two-way finite automata: Old and recent results. Fundam. Inform., 126(2-3):225-246, 2013.

[13] Michael O. Rabin and Dana Scott. Finite automata and their decision problems. IBM J. Res. Dev., 3(2):114-125, April 1959.

[14] Christophe Reutenauer. Sur les éléments inversibles de l'algèbre de Hadamard des séries rationnelles. Bull. Soc. math. France, 110:225-232, 1982.

[15] Marcel-Paul Schützenberger. On the definition of a family of automata. Inform. and Control, 4:245-270, 1961.

[16] John C. Shepherdson. The reduction of two-way automata to one-way automata. IBM J. Res. Dev., 3(2):198-200, April 1959. 